DOI: $10.1002 /($ please add manuscript number $))$

Article type: Communication

\title{
A Facet-Specific Quantum Dot Passivation Strategy for Colloid Management and Efficient Infrared Photovoltaics
}

Younghoon Kim,,$^{\dagger}$ Fanglin Che,,$^{\dagger}$ Jea Woong Jo,,$^{\dagger}$ Jongmin Choi,,$^{\dagger}$ F. Pelayo García de Arquer, Oleksandr Voznyy, Bin Sun, Junghwan Kim, Min-Jae Choi, Rafael Quintero-Bermudez, Fengjia Fan, Chih Shan Tan, Eva Bladt, Grant Walters, Andrew H. Proppe, Chengqin Zou, Haifeng Yuan, Sara Bals, Johan Hofkens, Maarten B. J. Roeffaers, Sjoerd Hoogland, and Edward H. Sargent

Dr. Younghoon Kim, Dr. Fanglin Che, Dr. Jea Woong Jo, Dr. Jongmin Choi, Dr. F. Pelayo García de Arquer, Dr. Oleksandr Voznyy, Dr. Bin Sun, Dr. Junghwan Kim, Dr. Min-Jae Choi, Rafael Quintero-Bermudez, Dr. Fengjia Fan, Dr. Chih Shan Tan, Grant Walters, Andrew H. Proppe, Chengqin Zou, Dr. Haifeng Yuan, Dr. Sjoerd Hoogland, Prof. Edward H. Sargent*

Department of Electrical and Computer Engineering, University of Toronto, 10 King's College Road,

Toronto, Ontario, M5S 3G4, Canada

*E-mail:ted.sargent@utoronto.ca

Dr. Eva Bladt, Prof. Sara Bals

Electron Microscopy for Materials Science (EMAT),

University of Antwerp, Groenenborgerlaan 171,

B-2020 Antwerp, Belgium

Dr. Haifeng Yuan, Prof. Johan Hofkens

Departement of Chemistry,

KU Leuven, Celestijnenlaan 200F,

3001 Heverlee, Belgium

Prof. Maarten B. J. Roeffaers

Center for Surface Chemistry and Catalysis, Faculty of Bioscience Engineering,

KU Leuven, 3001 Heverlee, Belgium

Dr. Younghoon Kim

Convergence Research Center for Solar Energy

Daegu Gyeongbuk Institute of Science and Technology (DGIST)

Daegu 72988, Republic of Korea

Keywords: Colloidal quantum dots, narrow bandgap, infrared solar cells, sodium acetate, facet-specific passivation

Abstract: Colloidal nanocrystals combine size- and facet-dependent properties with solution processing. They offer thus a compelling suite of materials for technological 
applications. Their size- and facet-tunable features have been studied in synthesis; however, to exploit their features in optoelectronic devices, it will be essential to translate control over size and facets from the colloid all the way to the film. Largerdiameter colloidal quantum dots (CQDs) offer the attractive possibility of harvesting infrared (IR) solar energy beyond absorption of silicon photovoltaics. These CQDs exhibit facets (nonpolar (100)) undisplayed in small-diameter CQDs; and the materials chemistry of smaller nanocrystals fails consequently to translate to materials for the short-wavelength IR regime. We demonstrate a new colloidal management strategy targeting the passivation of both (100) and (111) facets using using distinct choices of cations and anions. The approach leads to narrow-bandgap CQDs with impressive colloidal stability and photoluminescence quantum yield. Photophysical studies confirm a reduction both in Stokes shift $(\sim 47 \mathrm{meV})$ and Urbach tail $(\sim 29 \mathrm{meV})$. Our approach provides a $\mathbf{\sim 5 0 \%}$ increase in the power conversion efficiency of IR photovoltaics compared to controls, and a $\sim 70 \%$ external quantum efficiency at their excitonic peak.

Colloidal nanocrystals including metals, semiconductors and ceramics have been developed through advances in surface and colloid chemistry, and have inspired intense interest in technological applications due to their size-dependent features and their ease of materials processing. ${ }^{[1-10]}$

The surface structure of colloidal nanocrystals is sensitive to the crystal size. The complex surface structure that emerges immediately following nanocrystal synthesis ${ }^{[11-13]}$ has seen extensive study; but is underexplored in the post-synthetic processes responsible for assembling colloidal nanocrystals into semiconductor thin films.

Lead chalcogenide narrow-bandgap colloidal quantum dots (CQDs) enable harvesting of infrared (IR) light in single- and multi-junction thin-film solar cells, since their bandgaps can readily be tuned across the solar spectrum by controlling nanocrystal size. ${ }^{[14-21]}$ Advances 
in the surface chemistry of colloidal nanomaterials have enabled significant progress in the synthesis $^{[22-29]}$ and surface modification ${ }^{[30-36]}$ of IR CQDs. Recently, lead halide-based solution-phase ligand exchanges on CQDs having a $1.3 \mathrm{eV}(\sim 950 \mathrm{~nm})$ bandgap resulted in the highest-performing CQD solar cells to date, with certified power conversion efficiencies (PCEs) reaching 12\% under simulated AM1.5 full solar illumination. ${ }^{[33,34]}$ These advances were enabled by a careful size control and surface passivation in the solution phase; however, these new strategies have so far failed to translate to IR applications that rely on largerdiameter dots (i.e. $>3.5 \mathrm{~nm})$.

As the CQD size increases, the portion of nonpolar (100) facets with low surface energy increases at the expense of cation-rich polar (111) facets. ${ }^{[27,29]}$ Oleic acid is more weakly bound to nonpolar (100) facets compared to (111) facets: ${ }^{[29]}$ indeed it detaches readily from the (100) facet in polar solvents. This property, exploited in large-diameter CQDs to form locally-ordered superstructures via (100) facet assembly, ${ }^{[37-40]}$ produces CQD aggregation during solution-phase colloid management. We posited that this could underlie the failure of the best-performing previously-developed solution-phase ligand exchanges to enable high-quality longer-wavelength semiconductor solids.

We report herein a strategy to preserve colloidal stability and quantum confinement in narrow-bandgap CQDs during the solution-phase ligand exchange. We sought nonpolar (100) facet passivation in large-diameter CQDs by first replacing the ammonium cation $\left(\mathrm{NH}_{4}{ }^{+}\right)$used in conventional ligand exchange with alkaline metal cations: this enabled much better solubilization of narrow-bandgap CQDs in polar solvents during the ligand exchange. ${ }^{[33,34]}$ In light of the ionic sizes of the alkaline cations, taken together with the dissociation constant of alkaline metal acetate, we focused on sodium acetate $\left(\mathrm{Na}^{+} \cdot \mathrm{Ac}^{-}\right)$, which has the appropriate ionic size as well as a high dissociation constant. ${ }^{[41]}$ We hypothesized that this strategy could provide facet-specific passivation via sodium $(\mathrm{Na})$ and lead halides on the (100) and (111) facets of CQDs, respectively. Na-passivation of the (100) facet enables improved colloidal 
stability for narrow-bandgap CQDs in polar solvents, leading to superior photophysical properties.

The new strategy significantly enhanced solar cell performance, leading to $\sim 33 \%$ and $\sim 50 \%$ increases in PCEs under full and $1100 \mathrm{~nm}$-filtered solar spectra respectively, compared to the lead halide-only passivated CQDs fabricated using the conventional ligand exchange method. The best-performing devices showed record-high external quantum yields (EQEs) that reached $\sim 70 \%$ at the excitonic peak.

Schematic illustrations (Figure 1a) depict conventional and facet-specific solution ligand exchange for narrow-bandgap CQDs. CQDs with size-tunable bandgaps have a highsymmetry rock-salt structure and, when synthesized as nanocrystals using an oleic acid ligand, exhibit well-defined (111) and (100) facets (Figure S1). ${ }^{[27,29]}$ The surface of ultrasmall CQDs is dominated by the (111) facet, producing an octahedral nanoparticle shape. The (100) facet is expected to appear gradually with increasing CQD size, altering the (111) facet-only octahedron to the $(111) /(100)$ cuboctahedron shape. ${ }^{[27]}$ As-synthesized CQDs, stabilized by oleic acid ligands on the (100) and (111) facets through different binding mechanisms, are highly dispersible in nonpolar solvents such as octane. ${ }^{[29]}$

In previously-reported processing methods, the ligand exchange of oleic acid to lead halides (i.e., $\left[\mathrm{PbX}_{3}\right]^{-}$) occurs mainly on the Pb-rich and polar (111) facets of CQDs. This leads to a higher affinity of anionic ligand species with the aid of acidic $\mathrm{NH}_{4}{ }^{+}$cations. ${ }^{[33,38]}$ However, this method is not suited for narrow-bandgap CQDs exhibiting a fraction of larger (100) facets. Such CQDs are not colloidally stabilized, and thus aggregate and even fuse during ligand exchange as oleic acid ligands on (100) facets are easily removed in polar solvents such as dimethylformamide (DMF) (Figure 1a and 1b). ${ }^{[37,40]}$ As a result, ligandexchanged CQDs of large diameter show a brown turbid color in DMF solvent and a clumped structure: a symptom of degraded colloidal stability (Figure 1b and Figure S2). This indicates that the unpassivaed (100) facets contribute to CQD aggregation, corresponding to 
reduced colloidal stability in polar solvents. Decreased acess to surface sites during the exchange diminishes the extent to which lead halide ligands can be adsorbed onto the (111) facets during the solution-phase ligand exchange.

We targeted passivation of (100) facets to avoid aggregate assembly via unpassivated (100) facets in polar solvents that lead to CQD flocculation. By replacing the $\mathrm{NH}_{4}^{+} \cdot \mathrm{Ac}^{-}$with the $\mathrm{Na}^{+} \cdot \mathrm{Ac}^{-}$in a lead halide-based ligand exchange process, we pursued facet-specific passivation of CQDs with Na-passivated (100) facets and lead halide-passivated (111) facets, providing a higher degree of colloidal stability during and after the solution ligand exchange. This results in an increased presence of lead halide ligands on (111) facets due to the significantly reduced degree of aggregation during colloidal processing (Figure 1c). X-ray photoelectron spectroscopy (XPS) confirms that CQD solids prepared from the new passivation scheme exhibit an increased iodine signal associated with the $\mathrm{PbI}_{3}{ }^{-}$anion ligands, in addition to the presence of $\mathrm{Na}$. Each observation agrees with the overall evidence of improved passivation, and the higher iodine concentration agrees with the observation of more negative charges on CQD surfaces (Figure 1d, Figure S3 and Figure S4).

We examined the adsorption strength of $\mathrm{Na}^{*}$ and $\mathrm{NH}_{4}{ }^{*}$ on a $\mathrm{PbS}(100)$ surface using density functional theory (DFT) calculations. ${ }^{[42,43]}$ The differential charge density shows that electron exchange between $\mathrm{Na}^{*}$ and $\mathrm{PbS}(100)$ is more favorable compared to that between $\mathrm{NH}_{4}{ }^{*}$ and $\mathrm{PbS}(100)$. This suggests that the adsorption strength of $\mathrm{Na}^{*}$ on $\mathrm{PbS}(100)$ is stronger than that of $\mathrm{NH}_{4}{ }^{*}$ (Figure 2a). This corresponds well with adsorption energy $\left(\mathrm{E}_{\mathrm{ad}}\right)$ calculations for $\mathrm{Na}$ on $\mathrm{PbS}(100)$, which reveal a stronger binding of $\mathrm{Na}^{*}$ compared to $\mathrm{NH}_{4}{ }^{*}$ (Table S1). In addition, Na 1s core level binding energy shift (CLBEs) calculations ${ }^{[44]}$ alsong with experimental XPS results reveal $\mathrm{Na}^{+}$sits preferentialy on $\mathrm{PbS}(100)$ surfaces rather than on $\mathrm{PbS}(111)$. We found that $\mathrm{Na}$ binds to sulfur rather than lead on (100) facets, while in $\mathrm{PbS}(111)$, Na-adsorption occurs specifically on (111) facets passivated using iodine species. 
The CLBE of Na $1 s$ adsorbed on the iodine-covered (111) surface shifts only over the range of $-0.1 \mathrm{eV}$ to $0.0 \mathrm{eV}$ (with $\mathrm{Na}$ coverage: $1 / 9 \mathrm{ML}$ or $1 / 3 \mathrm{ML}$ ) relative to $\mathrm{Na}^{+} \cdot \mathrm{Ac}^{-}$salt reference (Figure $\mathbf{2 b}$ and Figure S5). In contrast, the CLBE of $\mathrm{Na} 1 s$ on (100) surface shifts over the range of $0.5 \mathrm{eV}$ to $0.8 \mathrm{eV}$ (with Na coverage: $1 \mathrm{ML}$ to $1 / 16 \mathrm{ML}$ ) (Figure S6). This is consistent with the Na $1 s$ binding energy shift of Na-passivated CQDs obtained from the XPS measurements, comparing with $\mathrm{Na} 1 s$ binding energy of $\mathrm{Na}^{+} \cdot \mathrm{Ac}^{-}$as a reference (Figure 2c). These results indicate that $\mathrm{Na}$ is concentrated primarily on the (100) facet. In addition, elemental analysis using inductively coupled plasma atomic emission spectrometry (ICPAES) reveals that Na remains on the CQDs after ligand exchange (Figure S7).

This new facet-specific passivation enabled narrow-bandgap CQDs to achieve a high solution-phase photoluminescence quantum yield (PLQY) in DMF (18\% PLQY). This is higher than that of lead halide-only exchanged CQDs (1.5\% PLQY), and close to the PLQY of original oleic acid-capped CQDs in octane (20\% PLQY) (Figure S8). Absorption and PL spectra reveal the effect of facet-specific passivation on the CQD solids, fabricated using the well-dispersed and dually-passivated CQDs. These show a narrower exciton peak with a fullwidth at half-maximum (FWHM) of $84 \mathrm{meV}$, compared to that of lead halide-only passivated CQDs (94 meV) having the same peak position of $1180 \mathrm{~nm}$. In addition, the PL spectrum shows that the FWHM of dually-passivated CQDs $(112 \mathrm{meV})$ is narrower than that of lead halide-only passivated CQDs (133 meV). The blue-shift of PL peak position reveals a smaller Stokes shift of $47 \mathrm{meV}$ for dually-passivated CQDs, compared to that of $62 \mathrm{meV}$ for lead halide-only passivated CQDs (Figure 3a). This indicates that the energetic disorder is minimized as a consequence of the significantly reduced degree of CQD aggregation, leading to improved surface passivation. ${ }^{[45,46]}$

To investigate the effect of energetic disorder in CQD solids in more detail, we examined optical absorption using photothermal deflection spectroscopy. This technique allows investigation of the tail states below the bandgap, allowing estimation of the Urbach 
energy. ${ }^{[47,48]}$ We found that CQD solids passivated using $\mathrm{Na}$ and lead halide showed an Urbach energy of $29 \pm 1 \mathrm{meV}$, which is $40 \%$ lower than that of lead halide-only passivated CQD solids (47 $\pm 2 \mathrm{meV})$ (Figure 3b).

Transient absorption (TA) spectroscopy revealed that dually-passivated CQDs exhibited a narrower bleach peak compared to lead halide-only passivated CQDs, indicating a reduced polydispersity and lowered enegetic disorder (Figure 3c). Gaussians fits of the TA spectra show a narrower FWHM of the excitonic bleaching signal of dually-passivated CQDs $(29 \pm 1 \mathrm{meV})$ vs. lead halide-only $(34 \pm 3 \mathrm{meV})$. Dually-passivated CQDs exhibit longer lifetimes $(\sim 27 \pm 2 \mathrm{~ns})$ compared to lead halide-only passivated CQDs ( $\sim 19 \pm 1 \mathrm{~ns})$ (Figure 3d). These photophysical studies indicate that the facet-specific passivation strategy enables reduced energetic disorder (i.e. a flattened energy landscape) of narrow-bandgap CQDs.

Field-effect transistor (FET) measurements revealed that the new protocol provides an approximately fourfold reduction in trap state density when dual passivation is used compared to the case of lead halide-only passivation (Figure S9). ${ }^{[49]}$ We carried out ultraviolet photoelectron spectra (UPS) measurements in order to investigate the energy band levels. Lead halide-only and dually-passivated CQDs showed no shift in the energy levels of conduction and valence bands (Figure S10).

We then pursued enhanced photovoltaic performance from narrow-bandgap CQDs prepared via the facet-specific passivation strategy. We fabricated solar cell devices using spin-coated layers of lead halide-exchanged CQDs with and without Na-passivation on (100). The device architecture consists of CQD photovoltaic layer deposited onto ZnO-coated ITO electrodes as an electron-transporting layer (ETL) and a thin layer of 1,2-ethanedithiol (EDT)treated CQDs (EDT-CQDs) as a hole-transporting layer (HTL) and gold deposition as the top metal electrode (Figure 4a). Current density-voltage $(J-V)$ curves for each device were acquired under AM1.5-simulatated full solar illumination (Figure 4b). The photovoltaic parameters of CQD solar cells made using the facet-specific passivation strategy are 
significantly improved, leading to an $8 \%$ increase in open-circuit voltage $\left(V_{\mathrm{OC}}\right)$, a $7 \%$ increase in $J_{\mathrm{SC}}$, a $14 \%$ increase in fill factor $(F F)$, and overall a $33 \%$ increase in PCE compared to the best-performing CQD solar cells fabricated using conventional lead halide-only passivation.

We measured the solar cell devices under filtered AM1.5 illumination mimicking the effect of absorption by a front wider-bandgap Si solar cells (i.e. beyond $1100 \mathrm{~nm}$ ). This allowed to examine light-harvesting capability in the IR region unharvested by silicon. The photovoltaic performance of each device after $1100 \mathrm{~nm}$ long-pass filter reveals that facetspecific passivation leads to a $13 \%$ increase in $V_{\mathrm{OC}}$, a $21 \%$ increase in $J_{\mathrm{SC}}$, a $6 \%$ increase in $F F$, and consequently a $48 \%$ increase in the $P C E$ of the best-performing devices, compared to the case of lead halide-only passivation (Figure 4c and Figure S11). Histograms of device performance are shown in Figure S12. Recently it has been reported that IR CQD solar cells fabricated operate efficiently, without need for UV-activation, on improved ZnO ETLs, an advance key to enabling the use of IR CQD solar cells as back cells in front-cell-filtered tandem photovoltaics. ${ }^{[50]}$

The EQE spectra further confirm the benefits of facet-specific passivation (Figure 4d). A significant EQE increase of dually-passivated CQDs beyond $1100 \mathrm{~nm}$ with an exciton peak of $\sim 70 \%$ is obtained, corresponding to a expected $J_{\mathrm{SC}}$ of $3.1 \pm 0.1 \mathrm{~mA} / \mathrm{cm}^{2}$. This is in contrast to lead halide-only passivated devices $\left(\mathrm{EQE}=50 \%\right.$ and $\left.J_{\mathrm{SC}}=2.5 \pm 0.1 \mathrm{~mA} / \mathrm{cm}^{2}\right)($ Figure S13). The expected $J_{\mathrm{SC}}$ of lead halide-only and dually-passivated CQD solar cell devices are measured to be $23.8 \pm 0.6$ and $25.7 \pm 0.4 \mathrm{~mA} / \mathrm{cm}^{2}$ for the full solar spectrum, in agreement with measured $J_{\mathrm{SC}}$ values under AM1.5.

We also characterized the photovoltaic performance of CQD solar cells based on lead halide-only passivated CQD inks prepared using different concentrations of $\mathrm{NH}_{4}^{+} \cdot \mathrm{Ac}^{-}$in the ligand exchange solution. As the amount of $\mathrm{NH}_{4}^{+} \cdot \mathrm{Ac}^{-}$increases, narrow-bandgap CQDs show phase-separation during the solution ligand exchange: more $\mathrm{NH}_{4}^{+}$in the polar solvent produces better stabilization of the colloidal phase. ${ }^{[30,33]}$ However, too large an amount of 
$\mathrm{NH}_{4}{ }^{+} \cdot \mathrm{Ac}^{-}$results in CQD fusion, which we attribute to the effect of $\mathrm{NH}_{4}^{+}$on the surface: the CQDs suffer from the elimination of $\mathrm{Pb}$-oleate from the surface and become are fused. ${ }^{[26,51,52]}$ As a result, photovoltaic parameters decrease with increased $\mathrm{NH}_{4}{ }^{+} \cdot \mathrm{Ac}^{-}$for the same thickness of active layer film (Figure S14). This agrees with the finding that absorption and PL spectra peaks become broader upon CQD fusion (Figure S15 and S16).

This work demonstrates facet-specific passivation implemented in a solution-phase ligand exchange process, enabling well-passivated and colloidally-stable CQD inks. $\mathrm{Na}$ cations selectively passivate nonpolar (100) facets that had previously been left unaddressed, leading to a facet-specific passivation on both polar (111) and nonpolar (100) facets in ligandexchanged CQDs. The approach enabled protection against CQD aggregation during ligand exchange, improved stability of the exchanged colloid and enhanced photophysical properties. The dually-passivated CQD solids prepared via the facet-specific strategy show improved solar cell performance under AM1.5 full solar spectrum (33\% increase in PCE) and beyond $1100 \mathrm{~nm}(48 \%$ increase in $P C E)$, with $\sim 70 \%$ EQE at the excitonic peak. Since the surface structure of colloidal nanocrystals varies with crystal size, the approach provides a means to achieve further improvements in nanocrystal-based devices.

\section{Experimental Section}

Preparation of PbS CQDs: Oleic-acid PbS CQDs (OA-CQDs) were synthesized using a previously published method. ${ }^{[28]}$ Dually-passivated PbS CQDs were prepared through a solution ligand exchange process of OA-CQDs in a test tube under atmospheric conditions as follows. First, for precursor solutions, $0.23 \mathrm{mg}(0.50 \mathrm{mmol})$ of lead iodide $\left(\mathrm{PbI}_{2}\right), 0.037 \mathrm{mg}$ $(0.11 \mathrm{mmol})$ of lead bromide $\left(\mathrm{PbBr}_{2}\right)$ and $0.017 \mathrm{mg}(0.21 \mathrm{mmol})$ of sodium acetate $\left(\mathrm{Na}^{+} \cdot \mathrm{Ac}^{-}\right)$ were completely dissolved in $5 \mathrm{~mL}$ of dimethylformamide (DMF). $5 \mathrm{~mL}$ of OA-CQDs dispersed in octane $\left(6 \mathrm{mg} \mathrm{mL}^{-1}\right)$ were added to the precursor solution, and transferred to DMF 
phase by vortexing vigorously for $5 \mathrm{~min}$. The PbS CQDs in DMF phase were then washed three times using octane in order to remove residual original ligands (i.e., OA). After being washed completely, the dually-passivated PbS CQDs were precipitated by adding toluene (2.5 $\mathrm{mL}$ ) as an anti-solvent, and dried under vacuum for $20 \mathrm{~min}$, and finally dispersed in a mix of $90 \%$ butylamine (BTA) and 10\% DMF at the desired concentrations. For the lead halide-only passivated $\mathrm{PbS}$ CQDs, we replaced $\mathrm{Na}^{+} \cdot \mathrm{Ac}^{-}$in the same recipe above mentioned with ammonium acetate. In this case, two phase CQD solution after ligand exchange is not clearly phase-separated, therefore it is forced by centrifugation to separate two phase for removing the remaining OA from DMF phase.

Measurement of high angle annular dark field scanning transmission electron microscopy (HAADF-STEM): High resolution HAADF-STEM images were acquired using a cubed FEI Titan microscope operating at $300 \mathrm{kV}$. A probe semiconvergence angle of $\sim 20 \mathrm{mrad}$ was used.

Measurement of X-ray photoelectron spectroscopy (XPS): XPS measurements were performed using a Thermo Scientific K-Alpha system, with a $50 \mathrm{eV}$ pass energy, and binding energy steps of $0.05 \mathrm{eV}$. All binding energy values were corrected relative to the reference $\mathrm{C}$ 1s peak $(284.5 \mathrm{eV})$. All signals were normalized to $\mathrm{Pb}$.

Measurement of optical absorption, photoluminescence $(P L)$ and photoluminescence quantum yield (PLQY): The total light absorption (A) was determined by $\mathrm{A}=1-\mathrm{R}-\mathrm{T}$, where $\mathrm{R}$ is the total reflectance measured from the glass side and $\mathrm{T}$ is the total transmittance through the back of solar cells ( $\mathrm{T}=0$ for devices with gold electrode). $\mathrm{R}$ and $\mathrm{T}$ were measured using a Perkin Elmer LAMBDA 950 spectrometer equipped with an integrating sphere. PL spectra and PLQY measurements were carried out using a Horiba FluoroLog-3 spectrofluorometer in reflection geometry under ambient conditions. Integrated sphere was used for obtaining 
solution-phase PLQY values. The sample was excited using a $633 \mathrm{~nm}$ pulsed laser diode $(<1$ ns). The emission was passed through a $1000 \mathrm{~nm}$ blaze grating monochromator (iHR320) and collected by an infrared photomultiplier tube.

Measurement of transient absorption (TA): A regeneratively amplified $\mathrm{Yb}: \mathrm{KGW}$ laser at a $5 \mathrm{kHz}$ repetition rate (Light Conversion, Pharos) was used to generated ultrafast pulses at $1030 \mathrm{~nm}$. The output of the laser passes through a beamsplitter, where one arm is sent to an optical parametric amplifier (Light Conversion, Orpheus) to generate photoexcitation (pump) pulses with a $\sim 350$-fs-duration, and the other arm is sent to a sapphire crystal to generate the NIR continuum spectrum. The pump and probe pulses are then both sent into a commercial TA spectrometer (Ultrafast, Helios). The probe pulse was delayed relative to the pump pulse using a delay stage permitting delays up to $8 \mathrm{~ns}$, and a chopper was used to block every other pump pulse. The signal after the sample is dispersed by a grating spectrograph (Ultrafast, Helios) and subsequently collected by a CCD camera. Lifetimes were fit using biexponential decays convoluted with a Gaussian to account for the Instrument Response Function (IRF). TA spectral slices were fit as a sum of one or more Gaussian peaks with negative coefficients for bleaching signals and positive coefficients for photo induced absorption signals.

Fabrication of CQD solar cell devices: ITO glass substrates were cleaned by soaking and sonicating sequentially in acetone, isopropyl alcohol, and deionized water. $\mathrm{ZnO}$ nanoparticles were synthesized as previously reported. The $\mathrm{ZnO}$ nanoparticle solution was spin-coated onto the cleaned ITO glass two times at $3000 \mathrm{rpm}$ for $30 \mathrm{sec}$, and subsequently dual and lead halide-only passivated CQDs in a mixture of 90\% BTA and 10\% DMF $(200 \mathrm{mg} / \mathrm{mL})$ were spin-coated at $2600 \mathrm{rpm}$ for $30 \mathrm{sec}$. For the final device fabrication, two thin layers of 1,2ethanedithiol (EDT)-treated CQDs (EDT-CQDs) were deposited, followed by gold deposition as a top metal electrode. 


\section{Supporting Information}

Supporting Information is available from the Wiley Online Library or from the author.

\section{Acknowledgements}

$\dagger$ Y. Kim, F. Che, J. W. Jo, J. Choi contributed equally. This work was supported by King Abdullah University of Science and Technology (KAUST, Office of Sponsored Research (OSR), Award No. OSR-2017-CPF-3325), Ontario Research Fund-Research Excellence program (ORF7-Ministry of Research and Innovation, Ontario Research Fund-Research Excellence Round 7). E. B. and S. B gratefully acknowledge funding by the European Research Council (ERC Starting Grant Colouratom 335078). Y. Kim received financial support from the DGIST R\&D Programs of the Ministry of Science, ICT \& Future Planning of Korea (18-ET-01). The authors thank L. Levina, R. Wolowiec, D. Kopilovic, and E. Palmiano for their technical help over the course of this research.

Received: ((will be filled in by the editorial staff))

Revised: ((will be filled in by the editorial staff)) Published online: ((will be filled in by the editorial staff))

\section{References}

[1] V. F. Puntes, K. M. Krishnan, A. P. Alivisatos, Science 2001, 291, 2115.

[2] M.-C. Daniel, D. Astruc, Chem. Rev. 2004, 104, 293.

[3] X. Wang, J. Zhuang, Q. Peng, Y. Li, Nature 2005, 437, 121.

[4] J. Park, J. Joo, S. G. Kwon, Y. Jang, T. Hyeon, Angew. Chem. Int. Ed. 2007, 46, 4630.

[5] Y. Kim, C. Lee, I. Shim, D. Wang, J. Cho, Adv. Mater. 2010, 22, 5140.

[6] M. J. Polking, M.-G. Han, A. Yourdkhani, V. Petkov, C. F. Kisielowski, V. V. Volkov, Y. Zhu, G. Caruntu, A. P. Alivisatos, R. Ramesh, Nat. Mater. 2012, 11, 700.

[7] T. R. Gordon, M. Cargnello, T. Paik, F. Mangolini, R. T. Weber, P. Fornasiero, C. B. Murray, J. Am. Chem. Soc. 2012, 134, 6751.

[8] T.-H. Yang, K. D. Gilroy, Y. Xia, Chem. Sci. 2017, 8, 6730.

[9] F. Fan, O. Voznyy, R. P. Sabatini, K. T. Bicanic, M. M. Adachi, J. R. McBride, K. R. Reid, Y.-S. Park, X. Li, A. Jain, R. Quintero-Bermudez, M. Saravanapavanantham, 
M. Liu, M. Korkusinski, P. Hawrylak, V. I. Klimov, S. J. Rosenthal, S. Hoogland, E. H. Sargent, Nature 2017, 544, 75.

[10] V. L. Colvin, M. C. Schlamp, A. P. Alivisatos, Nature 1994, 370, 354.

[11] W. K Bae, J. Lim, D. Lee, M. Park, H. Lee, J. Kwak, K. Char, C. Lee, S. Lee, Adv. Mater. 2014, 26, 6387.

[12] X. Gong, Z. Yang, G. Walters, R. Comin, Z. Ning, E. Beauregard, V. Adinolfi, O. Voznyy, E. H. Sargent, Nat. Photonics 2016, 10, 253.

[13] G. Konstantatos, I. Howard, A. Fischer, S. Hoogland, J. Clifford, E. Klem, L. Levina, E. H. Sargent, Nature 2006, 442, 180.

[14] J.-S. Lee, M. V. Kovalenko, J. Huang, D. S. Chung, D. V. Talapin, Nat. Nanotechnol. 2011, 6, 348 .

[15] M. A. Hines, G. D. Scholes, Adv. Mater. 2003, 15, 1844.

[16] S. A. McDonald, G. Konstantatos, S. Zhang, P. W. Cyr, E. J. D. Klem, L. Levina, E. H. Sargent, Nat. Mater. 2005, 4, 138.

[17] I. Gur, N. A. Fromer, M. L. Geier, A. P. Alivisatos, Science 2005, 310, 462.

[18] C.-H. M. Chuang, P. R. Brown, V. Bulović, M. G. Bawendi, Nat. Mater. 2014, 13, 796.

[19] A. H. Ip, A. Kiani, I. J. Kramer, O. Voznyy, H. F. Movahed, L. Levina, M. M. Adachi, S. Hoogland, E. H. Sargent, ACS Nano 2015, 9, 8833.

[20] A. Kiani, B. R. Sutherland, Y. Kim, O. Ouellette, L. Levina, G. Walters, C.-T. Dinh, M. Liu, O. Voznyy, X. Lan, A. J. Labelle, A. H. Ip, A. Proppe, G. H. Ahmed, O. F. Mohammed, S. Hoogland, E. H. Sargent, Appl. Phys. Lett. 2016, 109, 183105.

[21] O. Ouellette, N. Hossain, B. R. Sutherland, A. Kiani, F. P. García de Arquer, H. Tan, M. Chaker, S. Hoogland, E. H. Sargent, ACS Energy Lett. 2016, 1, 852.

[22] I. Moreels, B. Fritzinger, J. C. Martins, Z. Hens, J. Am. Chem. Soc. 2008, 130, 15081. 
[23] A. Dong, X. Ye, J. Chen, Y. Kang, T. Gordon, J. M. Kikkawa, C. B. Murray, J. Am. Chem. Soc. 2011, 133, 998.

[24] J. Tang, K. W. Kemp, S. Hoogland, K. S. Jeong, H. Liu, L. Levina, M. Furukawa, X. Wang, R. Debnath, D. Cha, K. W. Chou, A. Fischer, A. Amassian, J. B. Asbury, E. H. Sargent, Nat. Mater. 2011, 10, 765.

[25] A. H. Ip, S. M. Thon, S. Hoogland, O. Voznyy, D. Zhitomirsky, R. Debnath, L. Levina, L. R. Rollny, G. H. Carey, A. Fischer, K. W. Kemp, I. J. Kramer, Z. Ning, A. J. Labelle, K. W. Chou, A. Amassian, E. H. Sargent, Nat. Nanotechnol. 2012, 7, 577.

[26] N. C. Anderson, M. P. Hendricks, J. J. Choi, J. S. Owen, J. Am. Chem. Soc. 2013, 135, 18536.

[27] H. Choi, J.-H. Ko, Y.-H. Kim, S. Jeong, J. Am. Chem. Soc. 2013, 135, 5278.

[28] Z. Ning, O. Voznyy, J. Pan, S. Hoogland, V. Adinolfi, J. Xu, M. Li, A. R. Kirmani, J. Sun, J. Minor, K. W. Kemp, H. Dong, L. Rollny, A. Labelle, G. Carey, B. Sutherland, I. Hill, A. Amassian, H. Liu, J. Tang, O. M. Bakr, E. H. Sargent, Nat. Mater. 2014, 13, 822.

[29] D. Zherebetskyy, M. Scheele, Y. Zhang, N. Bronstein, C. Thompson, D. Britt, M. Salmeron, A. P. Alivisatos, L.-W. Wang, Science 2014, 344, 1380.

[30] H. Zhang, J. Jang, W. Liu, D. V. Talapin, ACS Nano 2014, 8, 7359.

[31] R. W. Crisp, D. M. Kroupa, A. R. Marshall, E. M. Miller, J. Zhang, M. C. Beard, J. M. Luther, Sci.Rep. 2015, 5, 9945.

[32] X. Lan, O. Voznyy, A. Kiani, F. P. García de Arquer, A. S. Abbas, G.-H. Kim, M. Liu, Z. Yang, G. Walters, J. Xu, M. Yuan, Z. Ning, F. Fan, P. Kanjanaboos, I. Kramer, D. Zhitomirsky, P. Lee, A. Perelgut, S. Hoogland, E. H. Sargent, Adv. Mater. 2016, 28, 299.

[33] M. Liu, O. Voznyy, R. Sabatini, F. P. Garcia de Arquer, R. Munir, A. H. Balawi, X. Lan, F. Fan, G. Walters, A. R. Kirmani, S. Hoogland, F. Laquai, A. Amassian, E. H. Sargent, Nat. Mater. 2017, 16, 258. 
[34] J. Xu, O. Voznyy, M. Liu, A. R. Kirmani, G. Walters, R. Munir, M. Abdelsamie, A. H. Proppe, A. Sarkar, F. P. García de Arquer, M. Wei, B. Sun, M. Liu, O. Ouellette, R. QuinteroBermudez, J. Li, J. Fan, L. Quan, P. Todorovic, H. Tan, S. Hoogland, S. O. Kelley, M. Stefik, A. Amassian, E. H. Sargent, Nat. Nanotechnol. 2018, 13, 456.

[35] Y. Kim, K. Bicanic, H. Tan, O. Ouellette, B. R. Sutherland, F. P. García de Arquer, J. W. Jo, M. Liu, B. Sun, M. Liu, S. Hoogland, E. H. Sargent, Nano Lett. 2017, 17, 2349.

[36] H. Aqoma, M. A. Mubarok, W. T. Hadmojo, E.-H. Lee, T.-W. Kim, T. K. Ahn, S.-H. Oh, S.-Y. Jang, Adv. Mater. 2017, 29, 1605756.

[37] W. J. Baumgardner, K. Whitham, T. Hanrath, Nano Lett. 2013, 13, 3225.

[38] D. M. Balazs, D. N. Dirin, H.-H. Fang, L. Protesescu, G. H. ten Brink, B. J. Kooi, M. V. Kovalenko, M. A. Loi, ACS Nano 2015, 9, 11951.

[39] W. Walravens, J. D. Roo, E. Drijvers, S. ten Brinck, E. Solano, J. Dendooven, C. Detavernier, I. Infante, Zeger Hens, ACS Nano 2016, 10, 6861.

[40] J. J. Geuchies, C. van Overbeek, W. H. Evers, B. Goris, A. de Backer, A. P. Gantapara, F. T. Rabouw, J. Hilhorst, J. L. Peters, O. Konovalov, A. V. Petukhov, M. Dijkstra, L. D. A. Siebbeles, S. van Aert, S. Bals, D. Vanmaekelbergh. Nat. Mater. 2016, 15, 1248.

[41] A. Salis, B. W. Ninham, Chem. Soc. Rev. 2014, 43, 7358.

[42] G. Kresse, J. Hafner, Phys. Rev. B 1993, 47, 558.

[43] G. Kresse, J. Furthmüller, Comput. Mat. Sci. 1996, 6, 15.

[44] L. Köhler, G. Kresse, Phys. Rev. B 2004, 70, 165405.

[45] D. Pal, V. G. Stoleru, E. Towe, D. Firsov, Jpn. J. Appl. Phys. 2002, 41, 482.

[46] J. Gao, J. C. Johnson, ACS Nano 2012, 6, 3292.

[47] S. John, Phys. Rev. Lett. 1986, 57, 1777.

[48] D. Venkateshvaran, M. Nikolka, A. Sadhanala, V. Lemaur, M. Zelazny, M. Kepa, M. Hurhangee, A. J. Kronemeijer, V. Pecunia, I. Nasrallah, I. Romanov, K. Broch, I. McCulloch, D. Emin, Y. Olivier, J. Cornil, D. Beljonne, H. Sirringhaus, Nature 2014, 515, 384. 
[49] B. Sun, O Voznyy, H. Tan, P. Stadler, M. Liu, G. Wakters, A. H. Proppe, M. Liu, J. Fan, J. Li, M. Wei, J. Xu, Y. Kim, S. Hoogland, E. H. Sargent, Adv. Mater. 2017, 29, 170049. [50] J. Choi, J. W. Jo, F. P. García de Arquer, Y.-B. Zhao, B. Sun, J. Kim, M.-J. Choi, S.-W. Baek, A. H. Proppe, A. Seifitokaldani, D.-H. Nam, P. Li, O. Ouellette, Y. Kim, O. Voznyy, S. Hoogland, S. O. Kelly, Z.-H. Lu, E. H. Sargent, Adv. Mater. 2018, 30, 1801720.

[51] Y. Kim, E. Yassitepe, O. Voznyy, R. Comin, G. Walters, X. Gong, P. Kanjanaboos, A. F. Nogueira, E. H. Sargent, ACS Appl. Mater. Interfaces 2015, 7, 25007.

[52] E. Yassitepe, Z. Yang, O. Voznyy, Y. Kim, G. Walters, J. A. Castañeda, P. Kanjanaboos, M. Yuan, X. Gong, F. Fan, J. Pan, S. Hoogland, R. Comin, O. M. Bakr, L. A. Padilha, A. F. Nogueira, E. H. Sargent, Adv. Funct. Mater. 2016, 26, 8757. 

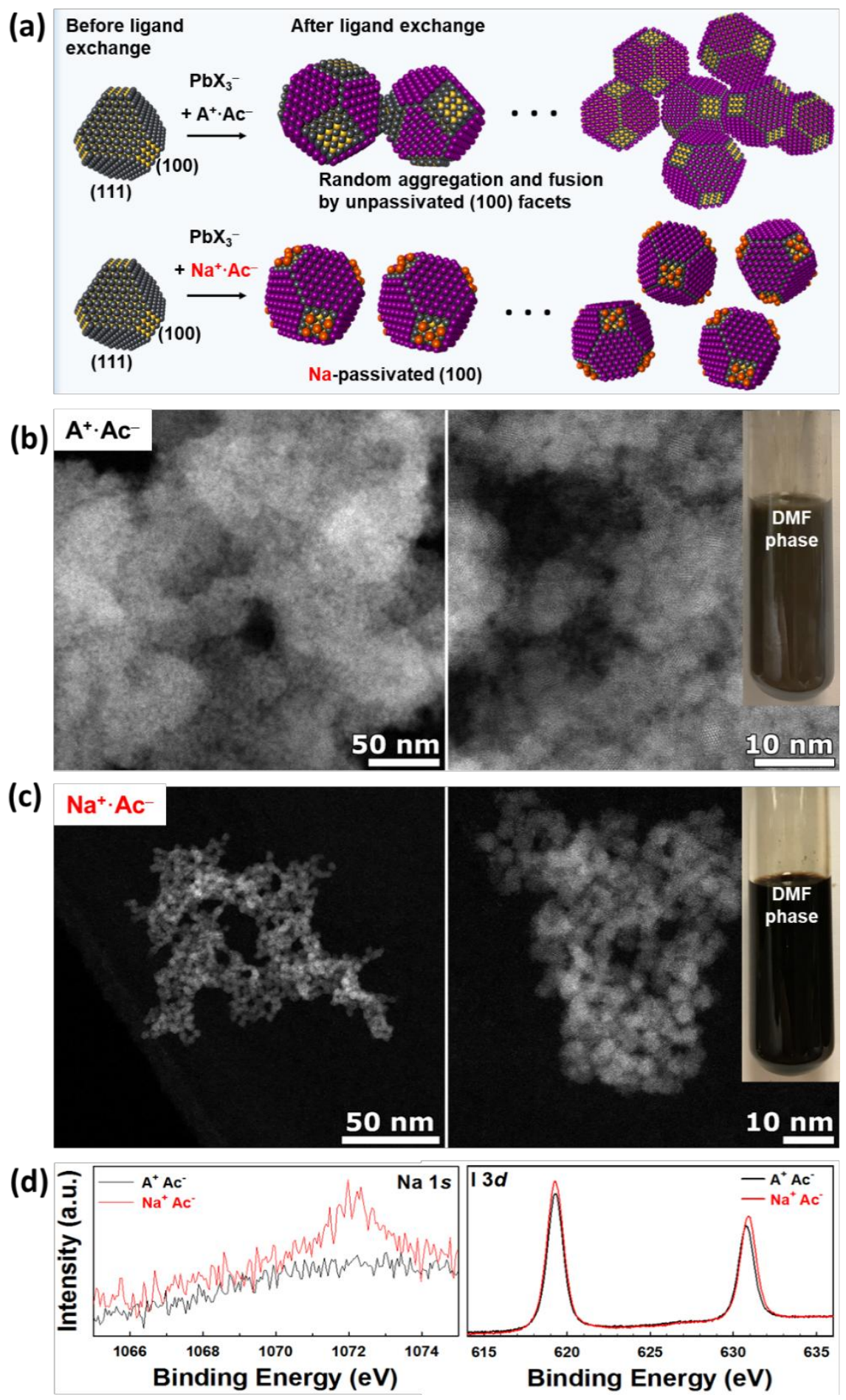

Figure 1. Lead halide-passivated CQDs with and without Na-passivation on the (100) facets. (a) Schematic illustrations of the conventional and facet-specific solution ligand exchanges for narrow-bandgap CQDs. HAADF-STEM images of (b) ligand-exchanged CQDs using the conventional method and (c) ligand exchanged CQDs using the facet-specific method. The photo images in (b) and (c) exhibit each CQD solution after solution-phase ligand exchange process (d) XPS results for $\mathrm{Na} 1 s$ and I $3 d$ peaks of each CQD after ligand exchange in solution phase. 
a
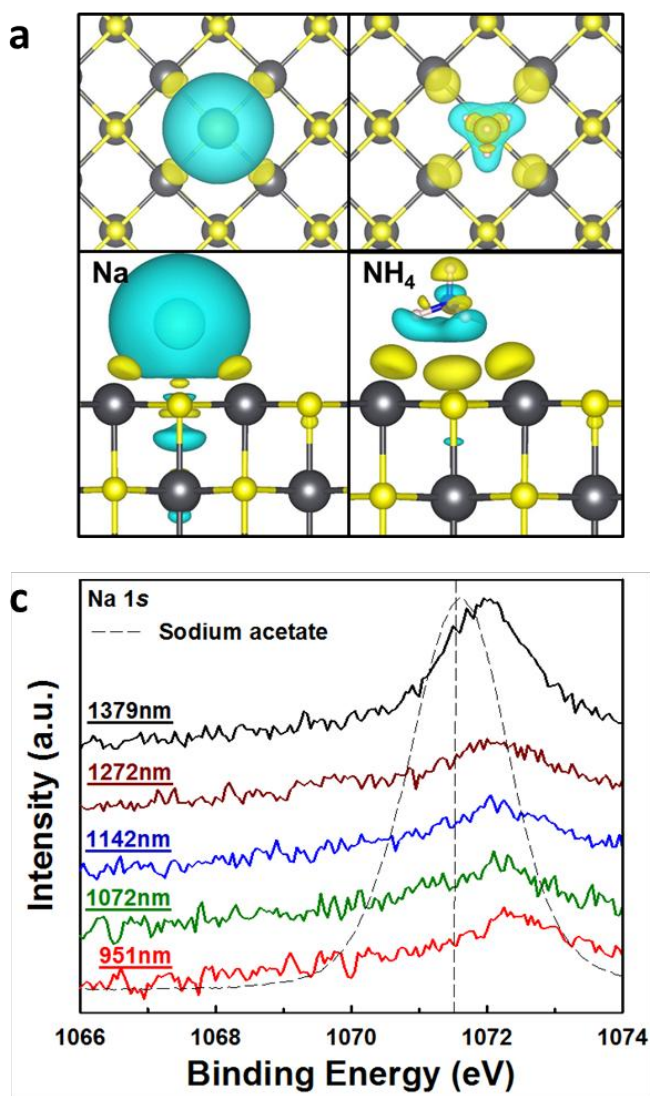
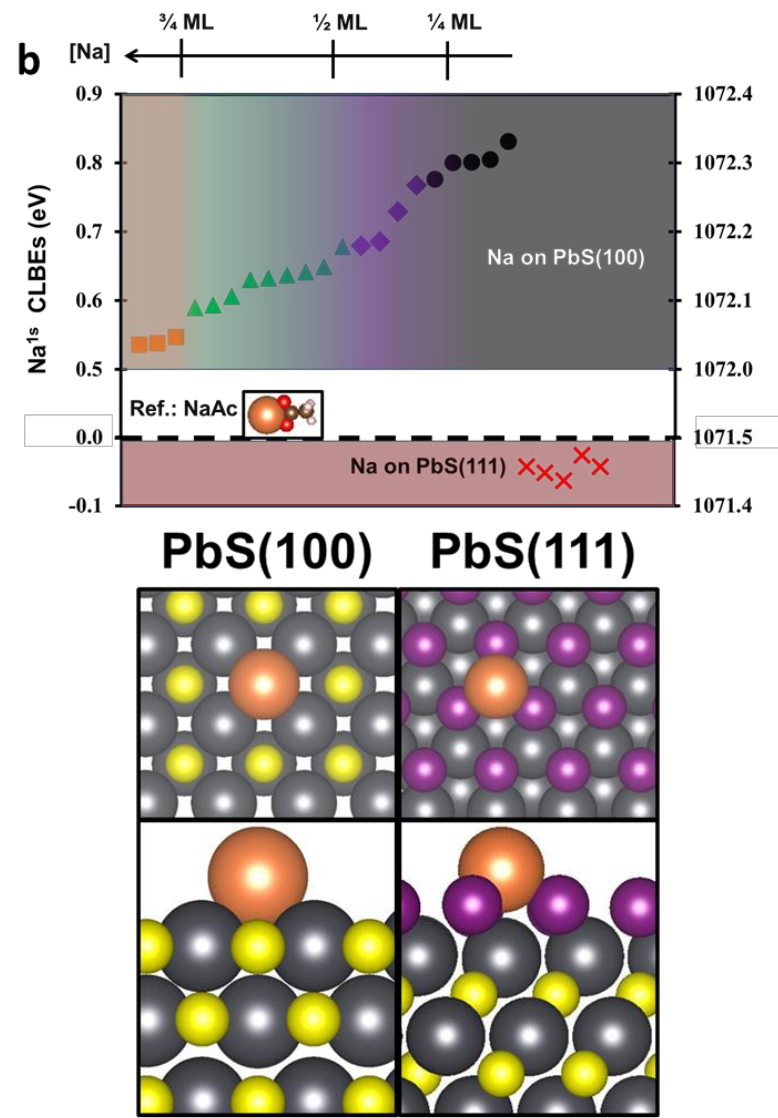

Figure 2. Effects of $\mathrm{Na}$ on $\mathrm{PbS}(100)$ facets. (a) Differential charge densities of $\mathrm{Na}^{+}$and $\mathrm{NH}_{4}{ }^{+}$on a $\mathrm{PbS}(100)$ surface with the coverage of $1 / 4$ monolayer (ML). The isosurface level of the differential charge densities is 0.001 electrons $/$ bohr $^{3}$, and the yellow or blue areas represent a gain or loss of electron, respectively. The $\mathrm{Na}, \mathrm{H}, \mathrm{N}, \mathrm{S}$, and $\mathrm{Pb}$ atoms are represented by the orange, pink, blue, yellow, and gray spheres, respectively. (b) The theoretical $\mathrm{Na} 1 s$ CLBEs for $\mathrm{Na}$ adsorbed on $\mathrm{PbS}(100)$ and $\operatorname{PbS}(111)$ with various $\mathrm{Na}$ coverage. All the $\mathrm{Na} 1 s$ CLBEs referred to the one in $\mathrm{Na}^{+} \cdot \mathrm{Ac}^{-}$. The $\mathrm{Na}, \mathrm{I}, \mathrm{S}$, and $\mathrm{Pb}$ atoms are represented by the orange, pink, yellow, and gray spheres, respectively. (c) $\mathrm{Na} 1 s$ binding energy shift of dually-passivated PbS CQDs with different bandgaps measured from XPS, comparing with the $\mathrm{Na} 1 s$ binding energy of $\mathrm{Na}^{+} \cdot \mathrm{Ac}^{-}$as a reference. 

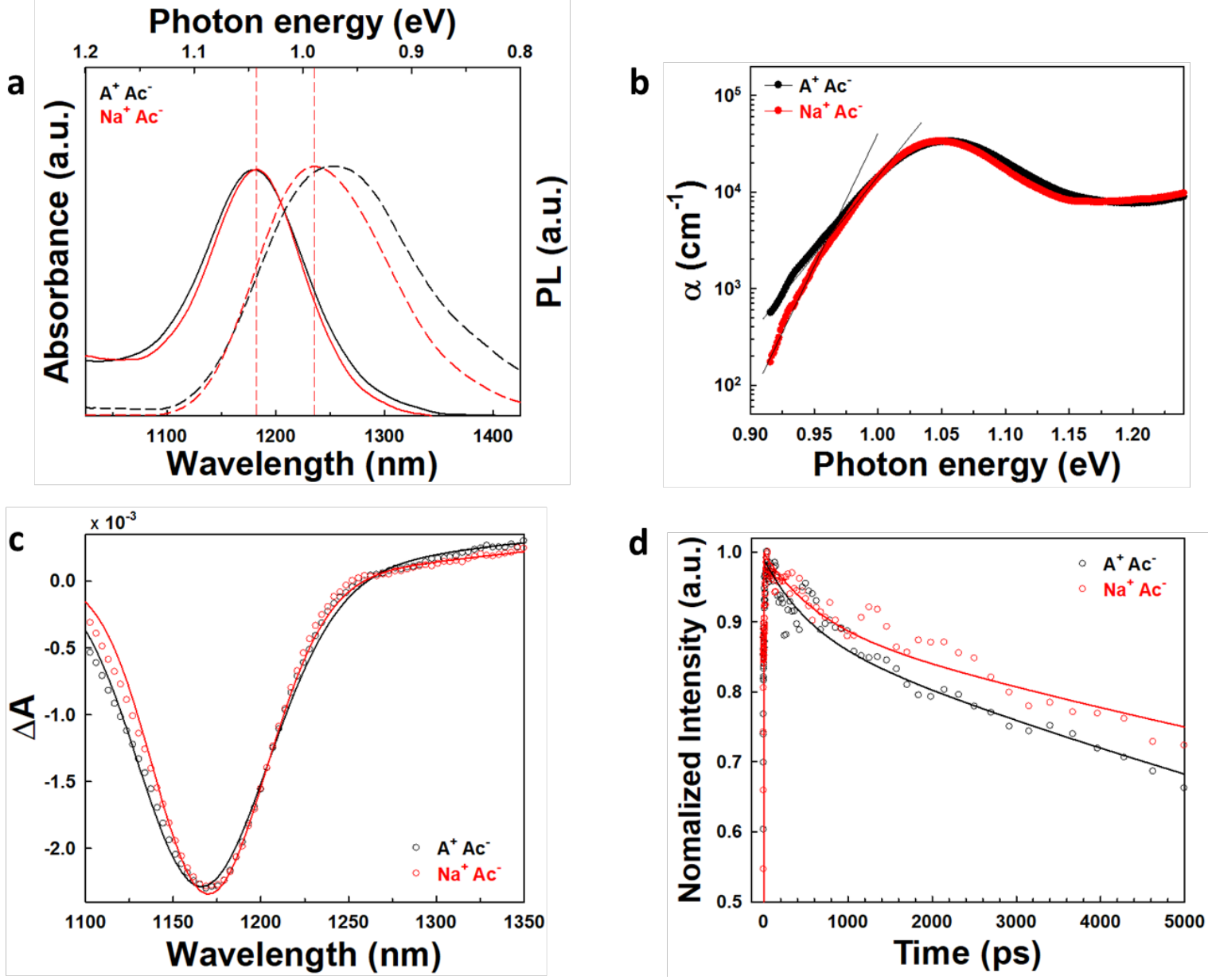

Figure 3. Photophysical studies on halide-passivated CQD solids with and without additional Na passivation. (a) Absorption and PL spectra of CQD solids prepared using $\mathrm{NH}_{4}^{+} \cdot \mathrm{Ac}^{-}$(black) and $\mathrm{Na}^{+} \cdot \mathrm{Ac}^{-}$(red), respectively. (b) Photothermal deflection spectroscopy measurements for each sample. The calculated Urbach energy is $28.5 \mathrm{meV}$ for the duallypassivated CQD solid using $\mathrm{Na}$ and lead halide (red), $46.7 \mathrm{meV}$ for the lead halide-only passivated CQD solid (black). (c) Excitonic bleaching peak with Gaussian fitting measured from TA. (d) Lifetimes at the central bleach with fits measured from TA. 
(a)
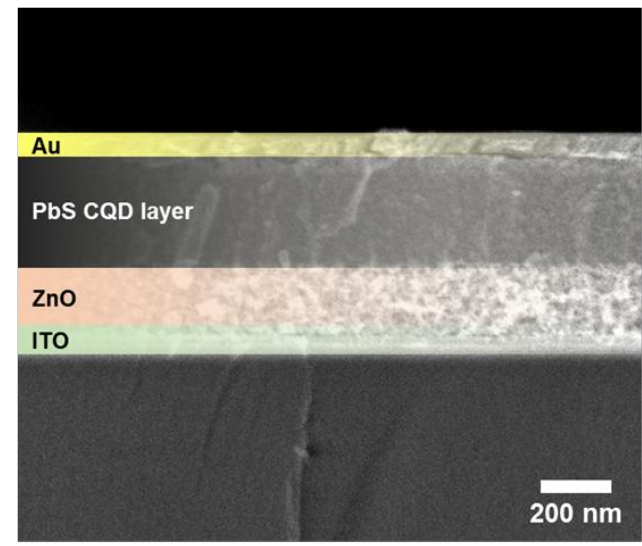

(c)

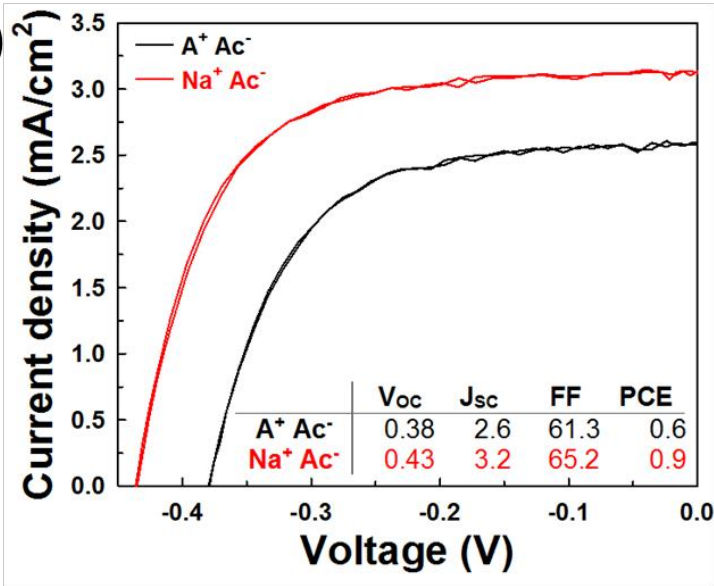

(b)

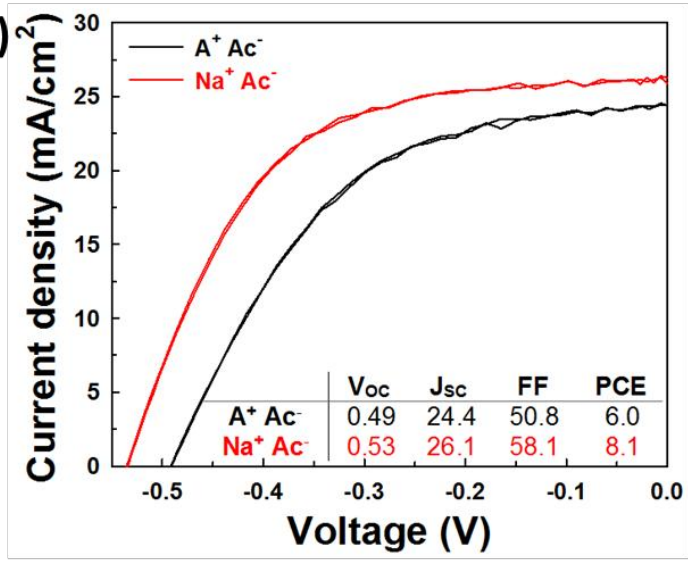

(d)

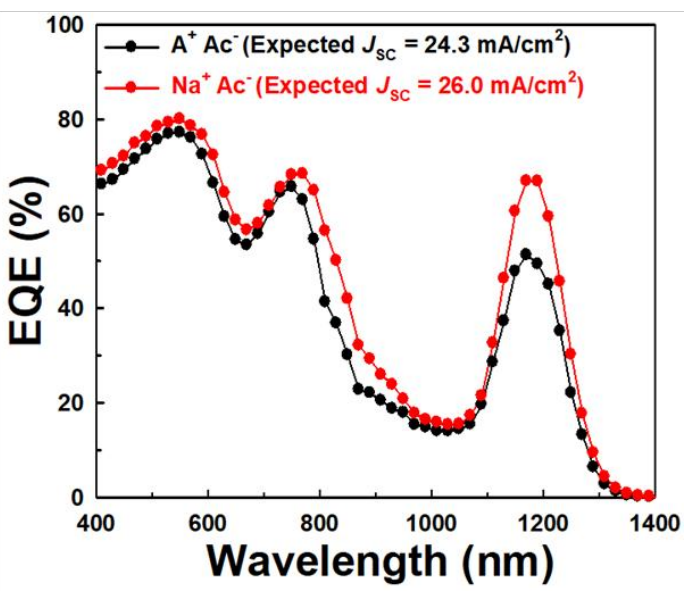

Figure 4. Halide-passivated CQD solar cell devices with and without Na-passivation on (100) facets. (a) Cross-sectional SEM image of an IR CQD solar cell device. Current-voltage $(J-V)$ characteristic of the lead halide-only and dually-passivated CQD solar cells (b) under AM1.5 simulated solar illumination, (c) after using a silicon solar cell representative filter (long-pass $1100 \mathrm{~nm}$ filter). (d) EQE spectra for each solar cell device measured from $400 \mathrm{~nm}$ to $1400 \mathrm{~nm}$. 
Table of contents entry

We report a novel solution-phase ligand exchange whereby (100) and (111) facets are selectively passivated by sodium and lead halides, respectively. This approach enables the realization of narrow-bandgap colloidal quantum dots with improved colloidal stability and enhanced photophysical properties. Our facet-specific passivation strategy significantly increases infrared (IR) solar cell performance, leading to a $50 \%$ increase in power conversion efficiency $(P C E)$ compared to the conventional lead halide-only passivation.

Keyword: Colloidal quantum dots, narrow bandgap, infrared solar cells, sodium acetate, facet-specific passivation

Younghoon Kim, ${ }^{\dagger}$ Fanglin Che ${ }^{\dagger}$ Jea Woong Jo, ${ }^{\dagger}$ Jongmin Choi, ${ }^{\dagger}$ F. Pelayo García de Arquer, Oleksandr Voznyy, Bin Sun, Junghwan Kim, Min-Jae Choi, Rafael QuinteroBermudez, Fengjia Fan, Chih Shan Tan, Eva Bladt, Grant Walters, Andrew H. Proppe, Chengqin Zou, Haifeng Yuan, Sara Bals, Johan Hofkens, Maarten B. J. Roeffaers, Sjoerd Hoogland, and Edward H. Sargent ${ }^{*}$

A Facet-Specific Quantum Dot Passivation Strategy for Colloid Management and Efficient Infrared Photovoltaics

\section{TOC figure}

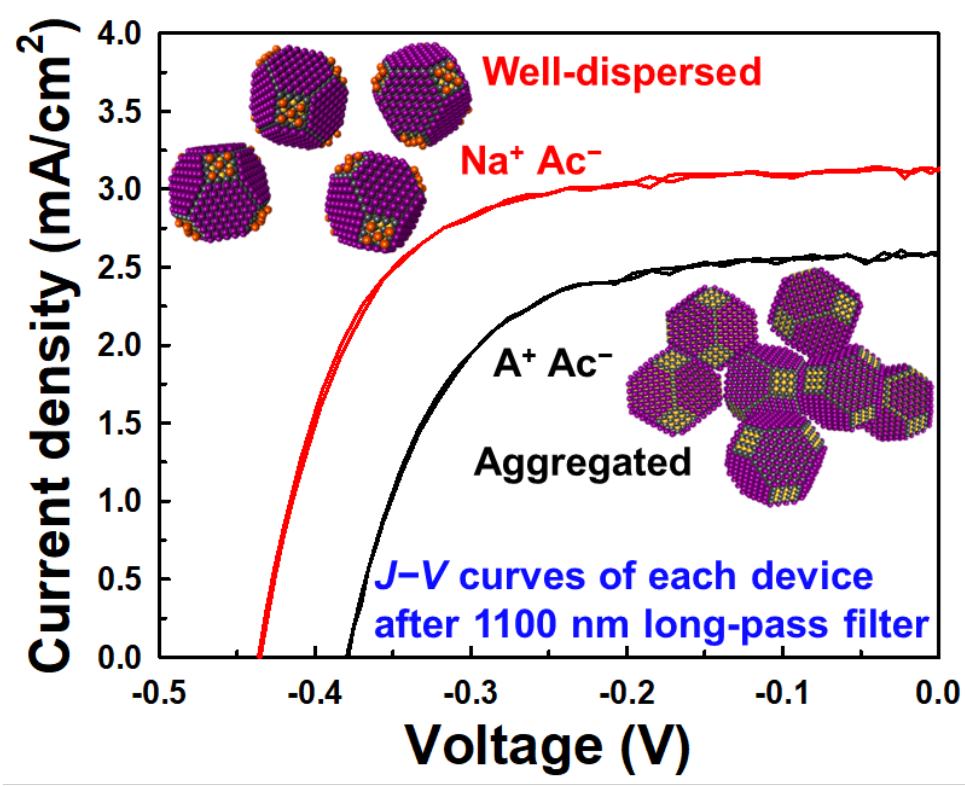




\section{Supporting Information}

\section{A Facet-Specific Quantum Dot Passivation Strategy for Colloid Management and Efficient Infrared Photovoltaics}

Younghoon Kim,,$^{\dagger}$ Fanglin Che, Jea Woong Jo, Jongmin Choi,,$^{\dagger}$ F. Pelayo García de Arquer, Oleksandr Voznyy, Bin Sun, Junghwan Kim, Min-Jae Choi, Rafael Quintero-Bermudez, Fengjia Fan, Chih Shan Tan, Eva Bladt, Grant Walters, Andrew H. Proppe, Chengqin Zou, Haifeng Yuan, Sara Bals, Johan Hofkens, Maarten B. J. Roeffaers, Sjoerd Hoogland, and Edward H. Sargent ${ }^{*}$

\section{Experimental Section}

Zeta potential measurements: Electrophoretic mobility was obtained using Zetasizer Nano-ZS (Malvern Intruments, Inc.) and with several scans of 100 runs in the high-resolution mode. Each CQD dispersed in DMF solvent was measured using a dip-cell setup with Pd electrodes.

Measurement of inductively coupled plasma atomic emission spectrometry (ICP-AES): Total contents of sodium and lead elements in the dually-passivated CQDs were characterized using the Optima 7300 DV ICP-AES (Perkin-Elmer, USA). The resulting CQDs were dissolved in 0.1 M nitric acid in deionized water for preparing ICP-AES samples.

Measurement of ultraviolet photoelectron spectroscopy (UPS): UPS measurement was carried out using a PHI5500 Multi-Technique system with $\mathrm{He} \mathrm{I}_{\alpha}$ radiation $(21.22 \mathrm{eV})$ from a discharge lamp. Each CQD solution was spin-coated onto gold substrates for the UPS measurement.

Field-effect transistor (FET) measurements: CQD-based FET devices were fabricated based on the bottom-gate and top-contact architectures. Ti $(70 \mathrm{~nm})$ and $\mathrm{ZrO}_{2}(15 \mathrm{~nm})$ were sequentially deposited onto the bare glass substrates and used as bottom-gate and dielectric 
layers in FET devices, respectively. Lead halide-only and dully-passivated CQDs were spincoated on the substrates, respectively, and finally Au was deposited as top-contact electrodes. The transfer curves of FET device were performed using Agilent 4155 semiconductor analyzer. Carrier mobility of FET devices was calculated using the equation (1) as follows:

Equation (1) : $I_{\mathrm{ds}}=\mu C_{\mathrm{i}}\left(V_{\mathrm{gs}}-V_{\mathrm{th}}\right) V_{\mathrm{ds}} W / L$

where $I_{\mathrm{ds}}$ is the drain current, $\mu$ is the carrier mobility in the linear regime, $C_{\mathrm{i}}$ is the gate dielectric capacitance per unit area $\left(450 \mathrm{nF} \mathrm{cm}{ }^{-2}\right), V_{\mathrm{gs}}$ is the gate voltage, $V_{\text {th }}$ is the threshold voltage, $L$ is the channel length $(50 \mu \mathrm{m})$, and $W$ is the channel width $(2500 \mu \mathrm{m})$. The trap state densities $\left(N_{t}\right)$ of each CQD were calculated using the transfer characteristic curves and the equation (2) as follows: ${ }^{1}$

Equation $(2): N_{t}=\left[\left(\frac{S \cdot e}{k T \cdot \ln (10)}-1\right) \cdot \frac{C_{i}}{e}\right]^{2} \cdot \varepsilon_{0} \varepsilon_{r}^{-1}$

Where $\mathrm{S}$ is the subthreshold wing, $k$ is the Boltzmann constant, $T$ is the temperature, $C_{i}$ is the capacitance of the insulating layer, $\varepsilon_{0}$ is the vacuum permittivity, $\varepsilon_{\mathrm{r}}$ is the electric constant of the film $(\sim 10.9)$.

Characterization of solar cell device: The current density-voltage curves of each device were tested under $\mathrm{N}_{2}$ flow using a Keithley 2400 SourceMeter. The solar spectrum at AM1.5G was simulated to within class A specifications ( $<25 \%$ spectral mismatch) with a xenon (Xe) lamp and filters (Solar Light Company Inc.) with measured intensity of $100 \mathrm{~mW} / \mathrm{cm}^{2}$. The illumination power was calibrated using a Melles-Griot broadband power meter and a Thorlabs broadband power meter through a circular optical aperture (area $0.049 \mathrm{~cm}^{2}$ ) at the position of the device and confirmed with a calibrated reference Si solar cell (Newport, Inc.). The final accuracy of the solar-to-electricity measurement was estimated to be $\pm 5 \% .1100 \mathrm{~nm}$ long-pass filter from Thorlabs, Inc. was used to represent a silicon solar cell. We used a 1100 
$\mathrm{nm}$ long-pass filter to emulate a silicon front cell and applied a correction factor to account for differences between the lamp and the full AM1.5 spectra.

Calculation of spectral mismatch: The spectral power within the wavelength region of interest was calibrated using the lamp spectrum supplied by the manufacturer. The absolute power of the lamp spectrum was measured with a calibrated power meter and a set of calibrated longpass filters. The external quantum efficiency (EQE) spectrum of the CQD solar cell device was measured and used to calculate the mismatch factor between the lamp spectrum filtered with a $1100 \mathrm{~nm}$ long-pass filter (1100 LPF) and the AM1.5 spectrum beyond $1100 \mathrm{~nm}$. The spectral mismatch was calculated with the ratio of the integral of the product of the lamp spectrum, the EQE spectrum, and 1100 LPF spectrum and the integral of the product of the AM1.5 spectrum, the EQE spectrum, and 1100 LPF spectrum. This resulted in a mismatch factor of 1.5. The main error in the mismatch factor is estimated to be in the calibration of the lamp spectrum, estimated to be about $10 \%$.

Measurement of external quantum efficiency (EQE): External quantum efficiency (EQE) spectra were carried out by aligning the cell to monochromatic light (a $400 \mathrm{~W}$ Xe lamp passing through a monochromator and proper cur-off filters). The active area was defined by optical aperture, and the light power was calibrated with Newport 818-UV and Newport 838IR photodetectors. The monochromatic light beam was chopped at $220 \mathrm{~Hz}$ and was collimated onto the device active layer using a solar simulator at 1 sun intensity to provide light bias. Pre-amplifier (Stanford Research Systems SR570) and lock-in amplifier (Stanford Research 830) were used for collecting the current signals from the solar cell devices. 
Other measurements: Cross-sectional images were obtained from the field-emission scanning electron microscope (FE-SEM, Hitachi SU8230). High-resolution transmission electron microscopy (HR-TEM) was performed using Hitachi HF3300. 

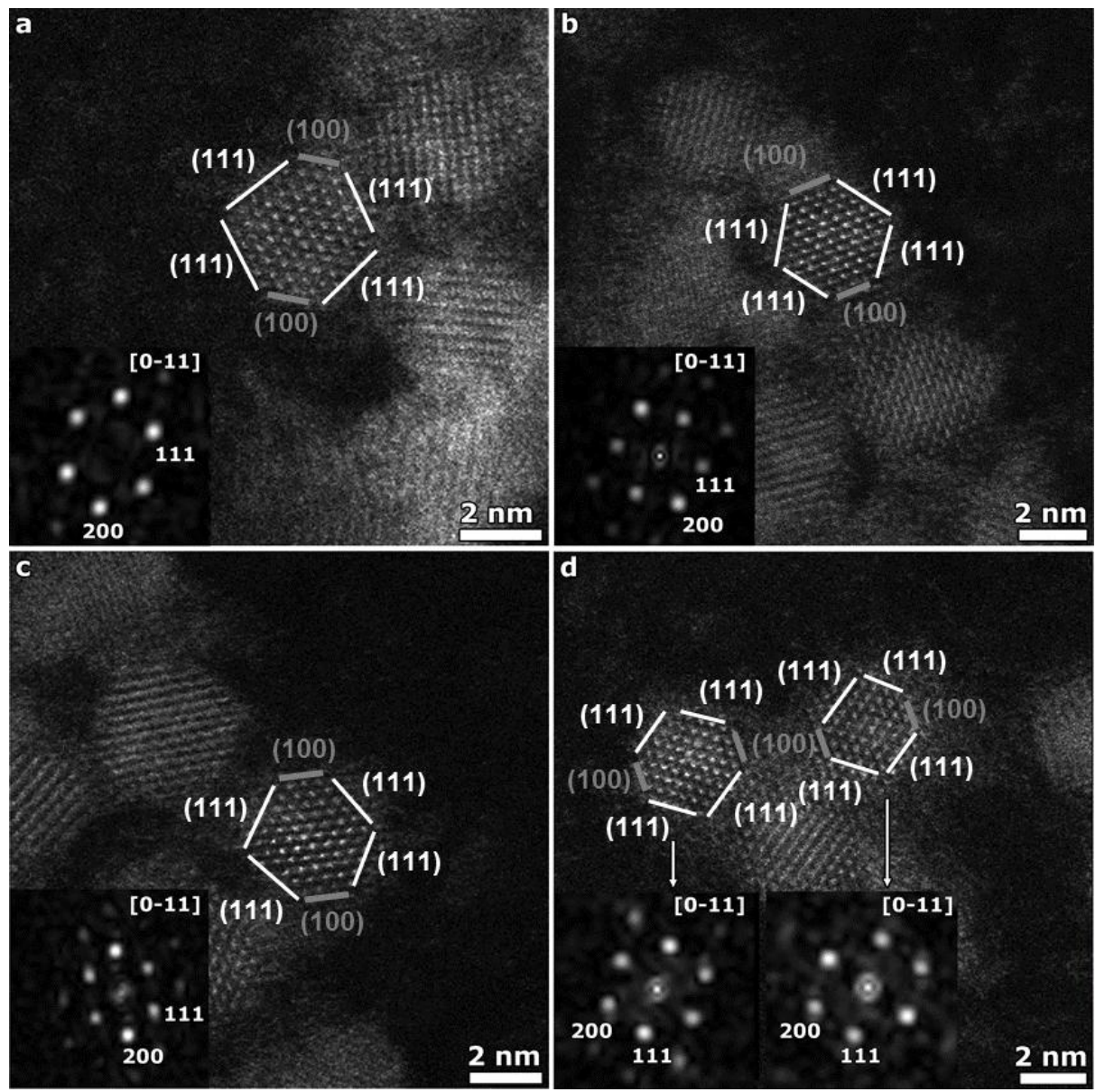

Figure S1. High resolution HAADF-STEM images and their corresponding diffractograms showing the presence of (111) and (100) facets of approximately $3.5 \mathrm{~nm}$-sized PbS CQDs used in this study. We investigated approximately $30 \mathrm{PbS}$ CQDs. 


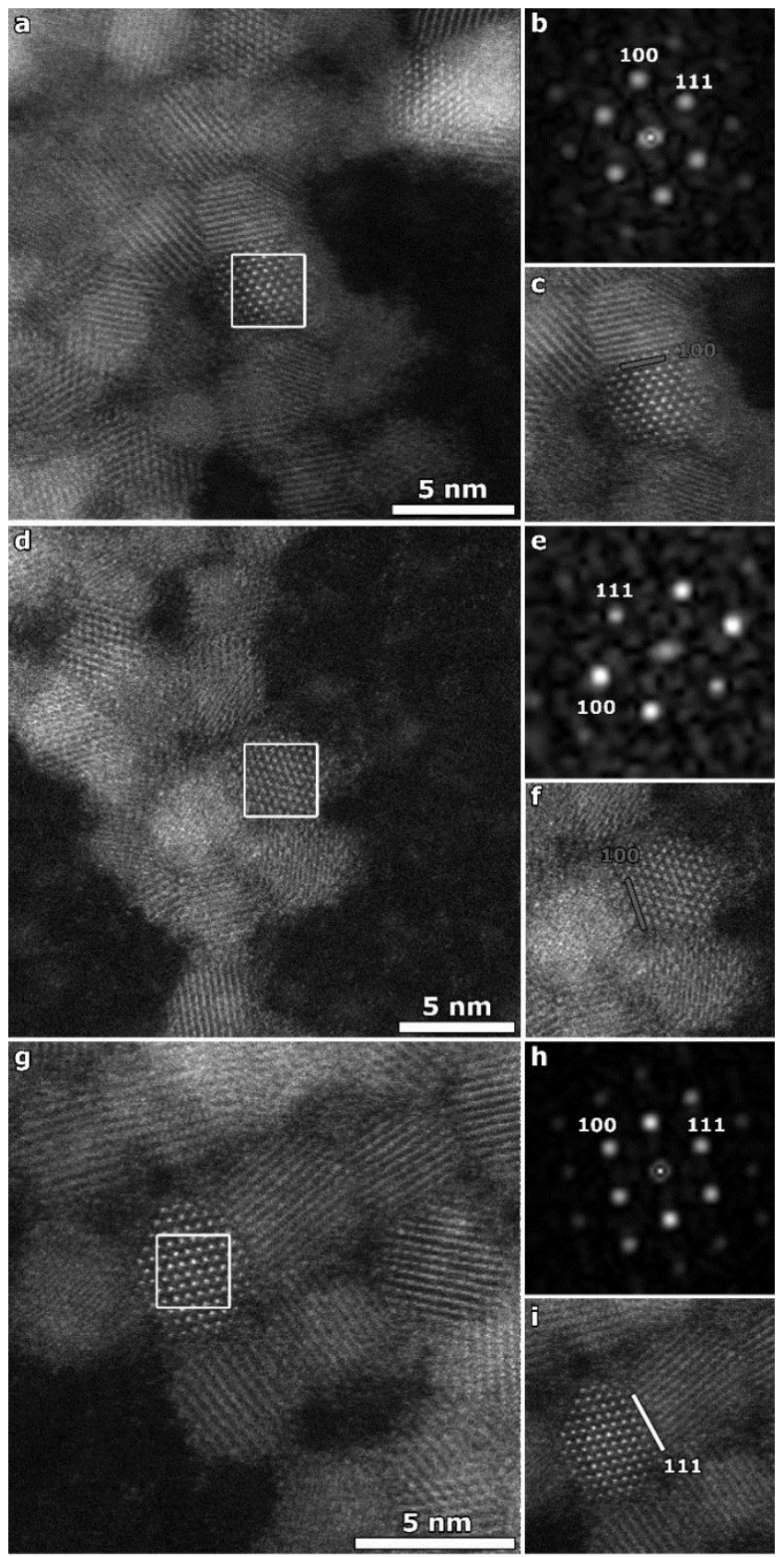

Figure S2. (a, d, g) High resolution HAADF-STEM images and (b, e, h) corresponding diffractograms (obtained at the location of the white rectangles in a, $\mathrm{d}$ and g) of lead halideonly passivated CQDs showing the CQD aggregation occurring along either the (100) facet (c, f) or the (111) facet (h). 

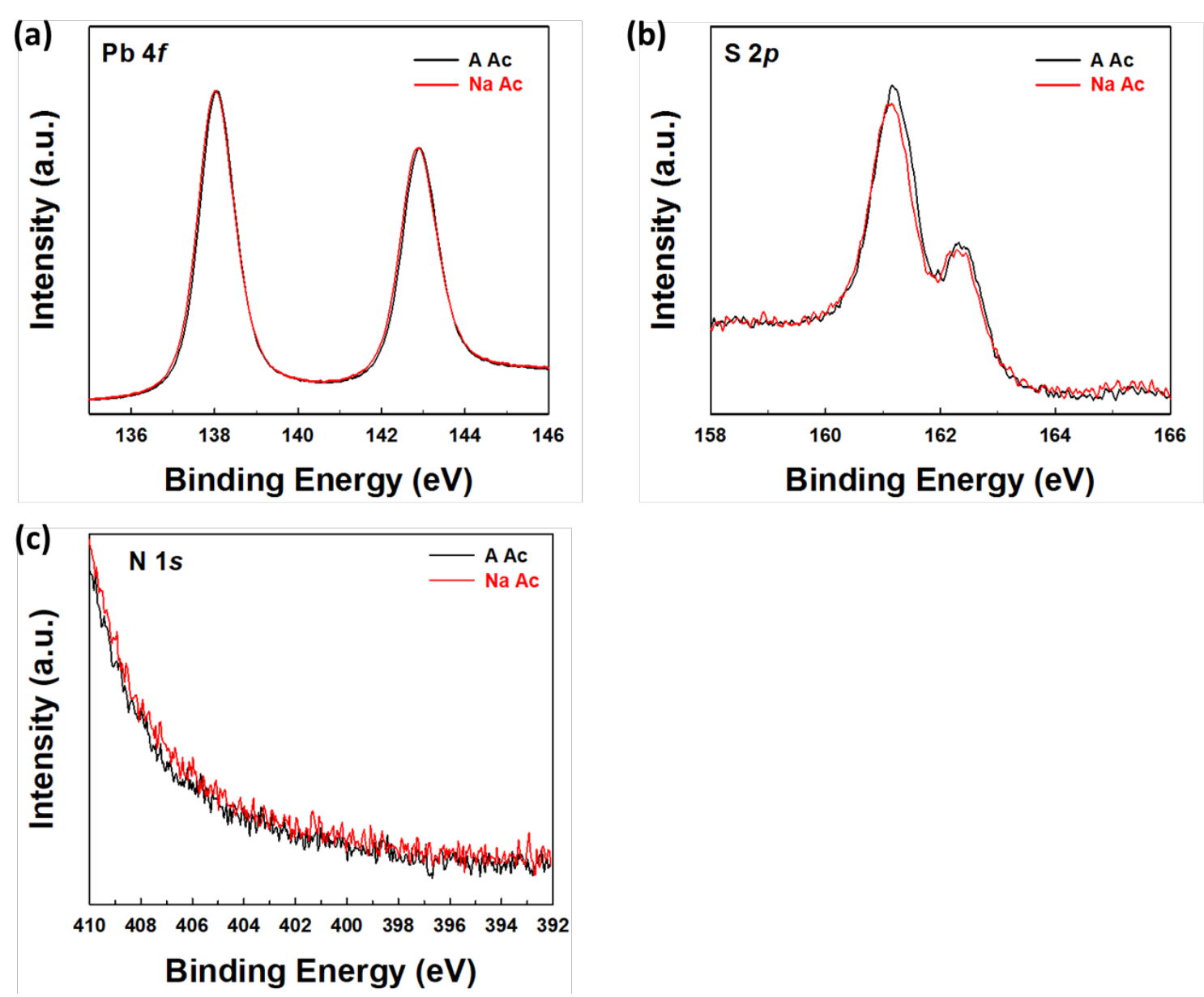

Figure S3. XPS results of lead halide-only and dually-passivated CQD solids after ligand exchange. (a) $\mathrm{Pb} 4 f$, (b) S $3 p$ and (c) $\mathrm{N} 1 s$. 


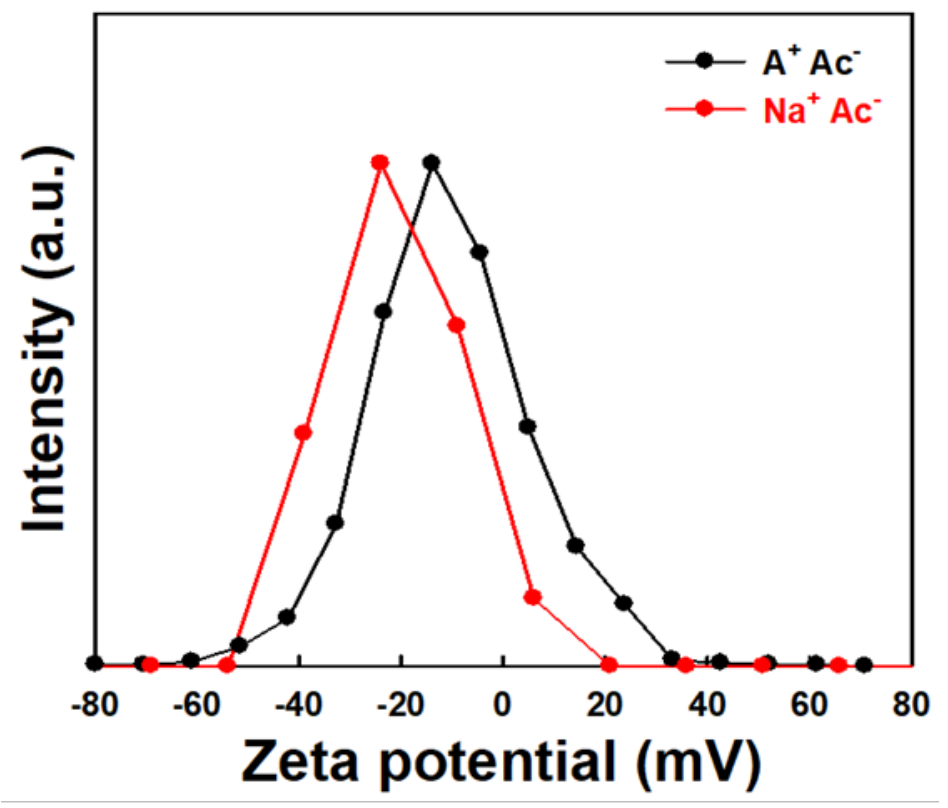

Figure S4. Zeta-potential results of lead halide-only and dully-passivated CQDs dispersed in DMF solvent. 
In the main manuscript, density functional theory (DFT) calculations were performed with the Vienna Ab Initio Simulation Package (VASP) code ${ }^{2,3}$. Perdew-Burke-Ernzerhof $(\mathrm{PBE})^{4}$ functionals were used to treat the exchange-correlation functional and the projectoraugmented wave (PAW) method ${ }^{5}$ was used to solve the ion-electron interactions in the periodic boundary conditions. A Monkhorst-Pack mesh ${ }^{6}$ with a grid of $(4 \times 4 \times 1) \mathbf{k}$-points was chosen for the surface calculations. The electronic wavefunctions at each k-point were expanded with a plan-wave energy cutoff up to $400 \mathrm{eV}$.

In this study, we chose the $\mathrm{PbS}(111)$ and (100) surfaces to investigate since they are the only exposed surfaces in a $\sim 3-4 \mathrm{~nm} \mathrm{PbS}$ QD. The $\mathrm{PbS}(111)$ and (100) surfaces were constructed using a $(3 \times 3)$ and a $(4 \times 4)$ periodicity with a four-layer slab separated by a vacuum layer of $31 \AA$. This can allow us to study the adsorbate coverage as low as 1/9 Monolayer (ML) on (111) facet and 1/16 ML on (100) facet, respectively. For the slabs, we fixed the bottom two layers at their bulk equilibrium positions and only relaxed the top two layers to examine the electronic interactions between the surface and adsorbates. The conjugate-gradient algorithm was adopted to relax the ions into their ground state with the length of the trial step controlled by a force scaling constant of 0.1. All self-consistent field (SCF) calculations were converged to $10^{-4} \mathrm{eV}$ and the forces were smaller than $0.03 \mathrm{eV} / \AA$. The PbS lattice constant was calculated and found to be $5.995 \AA(6.006 \AA)^{7}$ and is within $1.0 \%$ of the experimental value of $5.932 \AA^{8}$.

To examine the adsorption of sodium metal and ammonium cations on a $\mathrm{PbS}(100)$ surface, we first calculated the different adsorption sites (i.e., Pb-top, S-top, PbS-bridge). The optimized structure shows that the adsorption can only be stable when sodium adsorbs on the top of $\mathrm{S}$ atom on a $\mathrm{PbS}(100)$ surface. Additionally, according to the corresponding experiments, the $\mathrm{PbS}(111)$ surface is covered by the iodine species. Thus, for the $\mathrm{PbS}(111)$ surface, the sodium adsorbs at the hollow site on a $\mathrm{PbS}(111)$ surface with a full coverage of 
iodine species. The bonding strength of sodium and ammonia can be reflected by presenting the differential charge densities (as shown in Figure 2 in the main manuscript).

In addition, in the main manuscript, we performed the core level binding energy shift (CLBEs) calculations for $\mathrm{Na}$ adsorption on a $\mathrm{PbS}(100)$ and $\mathrm{PbS}(111)$ surface as compared to sodium acetate. The core level binding energy can present how tightly the core electrons of an atom are bound. The results can compare with the X-ray photoelectron spectroscopy (XPS) experimental measurement to confirm the binding environment for the adsorbate on a surface. DFT calculations with VASP code can examine the core level binding energy $\left(E_{C L B E}\right)$ by:

$E_{C L B E}=E_{n_{c}-1}-E_{n_{c}}$

where $E_{n_{c}-1}$ and $E_{n_{c}}$ stand for the total energy of the system where one core electron is removed and placed into the valence and the total energy of its corresponding ground state, respectively. However, the absolute DFT energies are not meaningful since VASP usually reports valence energies only. Thus, only the relative shifts of the core electron binding energies can be comparable with the experimental observations. The relative core level energy shifts are defined as:

$E_{C L B E s}=\left(E(\text { system } 1)_{n_{c}-1}-E(\text { system } 1)_{n_{c}}\right)-\left(E(\text { system } 2)_{n_{c}-1}-E(\text { system } 2)_{n_{c}}\right)$

In our work, we have calculated the core level binding energy shifts for the $\mathrm{Na} 1 \mathrm{~s}$ orbital when $\mathrm{Na}$ adsorb on a $\mathrm{PbS}(100)$ and $\mathrm{PbS}(111)$ surface. The reference in theoretical calculations is the $\mathrm{Na}$ 1s orbital in sodium acetate $\left(\mathrm{Na}^{+} \cdot \mathrm{Ac}^{-}\right)$, which is the same as experimental XPS measurement. The optimized configurations for the different coverages of the $\mathrm{Na}$ species on a $\mathrm{PbS}(111)$ surface covered with the iodine species are presented in Figure S5. The optimized configurations and absorption energies for the $\mathrm{Na}$ species on a $\mathrm{PbS}(100)$ surface with various coverages are presented in Figure S6 and Table S1. 


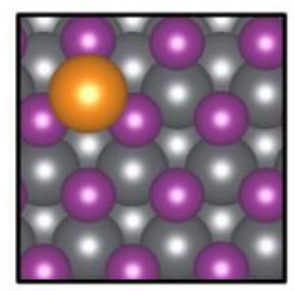

1/9 ML

$\mathrm{E}_{\text {CLBES }}=-0.04 \mathrm{eV}$

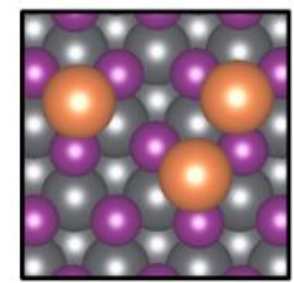

$1 / 3 \mathrm{ML}$

$\mathrm{E}_{\mathrm{CLBES}}=-\mathbf{0 . 0 3} \mathrm{eV}$

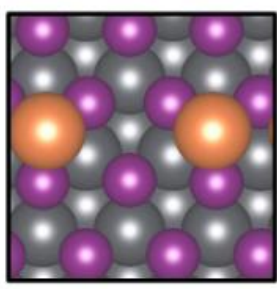

2/9 ML

$\mathrm{E}_{\mathrm{CLBES}}=-\mathbf{0 . 0 5} \mathrm{eV}$

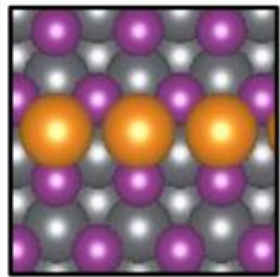

$1 / 3 \mathrm{ML}$

$\mathrm{E}_{\mathrm{CLBES}}=-\mathbf{0 . 0 3} \mathrm{eV}$

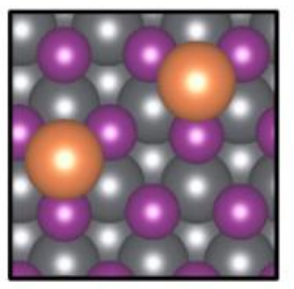

2/9 ML

$\mathrm{E}_{\text {CLBEs }}=-0.06 \mathrm{eV}$

Figure S5. The optimized configurations for the $\mathrm{Na}$ species on a $\mathrm{PbS}(111)$ surface covered with iodine species. The examined coverages for $\mathrm{Na}$ are in the range of $1 / 9 \mathrm{ML}$ to $1 / 3 \mathrm{ML}$.

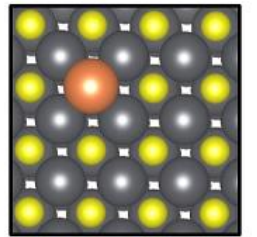

$1 / 16 \mathrm{ML}$

$\mathrm{E}_{\mathrm{CLBEs}}=0.78 \mathrm{eV}$

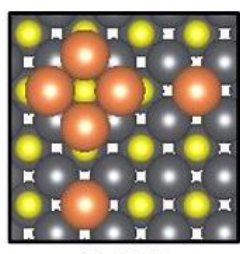

6/16 ML

$\mathrm{E}_{\text {CLBEs }}=0.77 \mathrm{eV}$

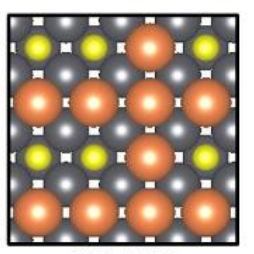

10/16 ML

$\mathrm{E}_{\text {CLBEs }}=0.64 \mathrm{eV}$

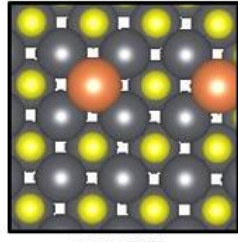

2/16 ML

$\mathrm{E}_{\mathrm{CLBES}}=0.80 \mathrm{eV}$

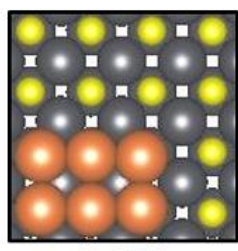

6/16 ML

$\mathrm{E}_{\text {CLBEs }}=0.68 \mathrm{eV}$

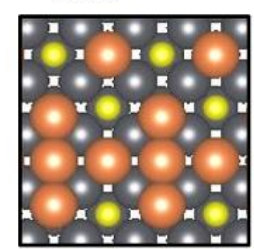

10/16 ML

$\mathrm{E}_{\text {CLBEs }}=0.59 \mathrm{eV}$

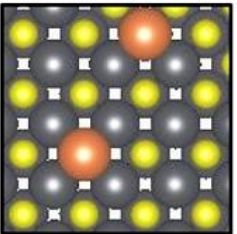

2/16 ML

$\mathrm{E}_{\mathrm{CLBES}}=0.80 \mathrm{eV}$

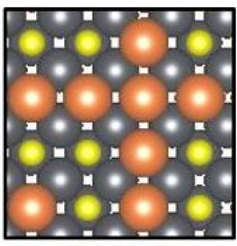

8/16 ML

$\mathrm{E}_{\text {CLBES }}=0.73 \mathrm{eV}$

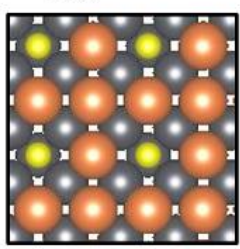

12/16 ML

$\mathrm{E}_{\text {CLBEs }}=0.54 \mathrm{eV}$

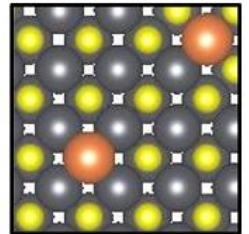

2/16 ML

$\mathrm{E}_{\text {CLBEs }}=0.80 \mathrm{eV}$

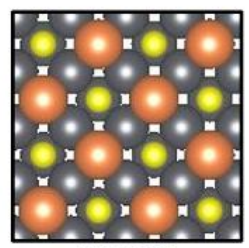

8/16 ML

$\mathrm{E}_{\mathrm{CLBES}}=0.69 \mathrm{eV}$

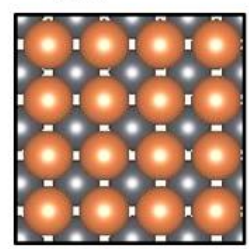

$1 \mathrm{ML}$

$\mathbf{E}_{\text {CLBEs }}=0.54 \mathrm{eV}$

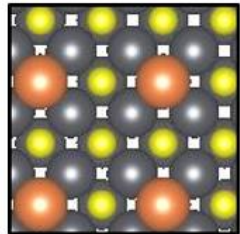

4/16 ML

$\mathrm{E}_{\mathrm{CLBES}}=0.80 \mathrm{eV}$

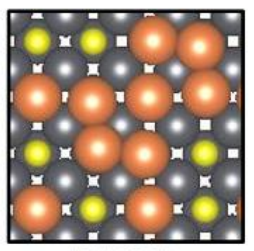

10/16 ML

$\mathrm{E}_{\text {CLBEs }}=0.65 \mathrm{eV}$

Figure S6. The optimized configurations for the $\mathrm{Na}$ species on a $\mathrm{PbS}(100)$ surface with various coverages in the range of $1 / 16$ Monolayer (ML) to $1 \mathrm{ML}$. 
Table S1. DFT calculations on the adsorption energy of $\mathrm{Na}^{*}$ on $\mathrm{PbS}(100)$ with various coverages.

\begin{tabular}{|c|c|}
\hline $\mathrm{Na}(\mathrm{ML})$ & $\mathrm{E}_{\mathrm{ad}}(\mathrm{eV})$ \\
\hline $1 / 16$ & -0.73 \\
\hline $2 / 16$ & -1.08 \\
\hline $4 / 16$ & -1.08 \\
\hline $6 / 16$ & -1.06 \\
\hline $8 / 16$ & -1.02 \\
\hline $10 / 16$ & -1.09 \\
\hline $12 / 16$ & -1.10 \\
\hline $16 / 16$ & -1.21 \\
\hline
\end{tabular}

Note: The adsorption energy of $\mathrm{Na}$ is calculated by $\mathrm{E}_{\mathrm{ad}}=\frac{E_{x N a_{P b S(100)}}-E_{P b S(100)}-x E_{N a}}{x}$. Here, $E_{x N a_{P b S(100)}}$ is the total energy of $\mathrm{Na}$ on a $\mathrm{PbS}(100)$ surface with $\mathrm{x} / 16 \mathrm{ML}$ coverage, $E_{P b S(100)}$ is the energy of a $\mathrm{PbS}(100)$ surface and $E_{N a}$ is the energy of one $\mathrm{Na}$ atom in a box. Similarly, the adsorption energy of $\mathrm{NH}_{4}$ on a $\mathrm{PbS}(100)$ is $-0.59 \mathrm{eV}$ at a coverage of $1 / 16 \mathrm{ML}$. 
(a)

\begin{tabular}{|c|c|c|c|c|}
\hline \multirow{2}{*}{$1142 \mathrm{~nm}$ CQDs } & \multicolumn{2}{|c|}{ Concentration $(\mathrm{ug} / \mathrm{mL})$} & \multicolumn{2}{c|}{ Atomic Ratio } \\
\cline { 2 - 5 } & $\mathrm{Na}$ & $\mathrm{Pb}$ & $\mathrm{Na}$ & $\mathrm{Pb}$ \\
\hline As-prepared & 5.11 & 100.2 & 0.0510 & 1 \\
\hline 1 wash with DMF & 5.10 & 99.1 & 0.0493 & 1 \\
\hline 2 wash with DMF & 5.13 & 100.0 & 0.0513 & 1 \\
\hline
\end{tabular}

(b)

\begin{tabular}{|c|c|c|c|c|}
\hline \multirow{2}{*}{ Sample } & \multicolumn{2}{|c|}{ Concentration $(\mathrm{ug} / \mathrm{mL})$} & \multicolumn{2}{c|}{ Atomic Ratio } \\
\cline { 2 - 5 } & $\mathrm{Na}$ & $\mathrm{Pb}$ & $\mathrm{Na}$ & $\mathrm{Pb}$ \\
\hline $1072 \mathrm{~nm}$ & 6.68 & 168.3 & 0.0397 & 1 \\
\hline $1142 \mathrm{~nm}$ & 5.11 & 103.6 & 0.0510 & 1 \\
\hline $1272 \mathrm{~nm}$ & 7.72 & 91.4 & 0.0845 & 1 \\
\hline
\end{tabular}

Figure S7. Elemental analysis was performed using ICP-AES to confirm the remaining sodium on the CQDs. (a) $1.08 \mathrm{eV}$ bandgap CQDs after facet-specific ligand exchange as a function of DMF washing times. For preparing the $1^{\text {st }}$ washed $1.08 \mathrm{eV}$ bandgap CQDs with DMF, as-prepared CQDs was redispersed into DMF, precipitated with toluene, and centrifuged. This process was repeated for $2^{\text {nd }}$ washed sample. As a result, sodium remained on the CQDs even though they were washed by DMF solvent. (b) Different bandgap CQDs with $1.16,1.08$ and $0.97 \mathrm{eV}$ after facet- specific ligand exchange. As the bandgap decrease, higher ratio of sodium to lead was obtained because of the increased (100)/(111) ratio. 


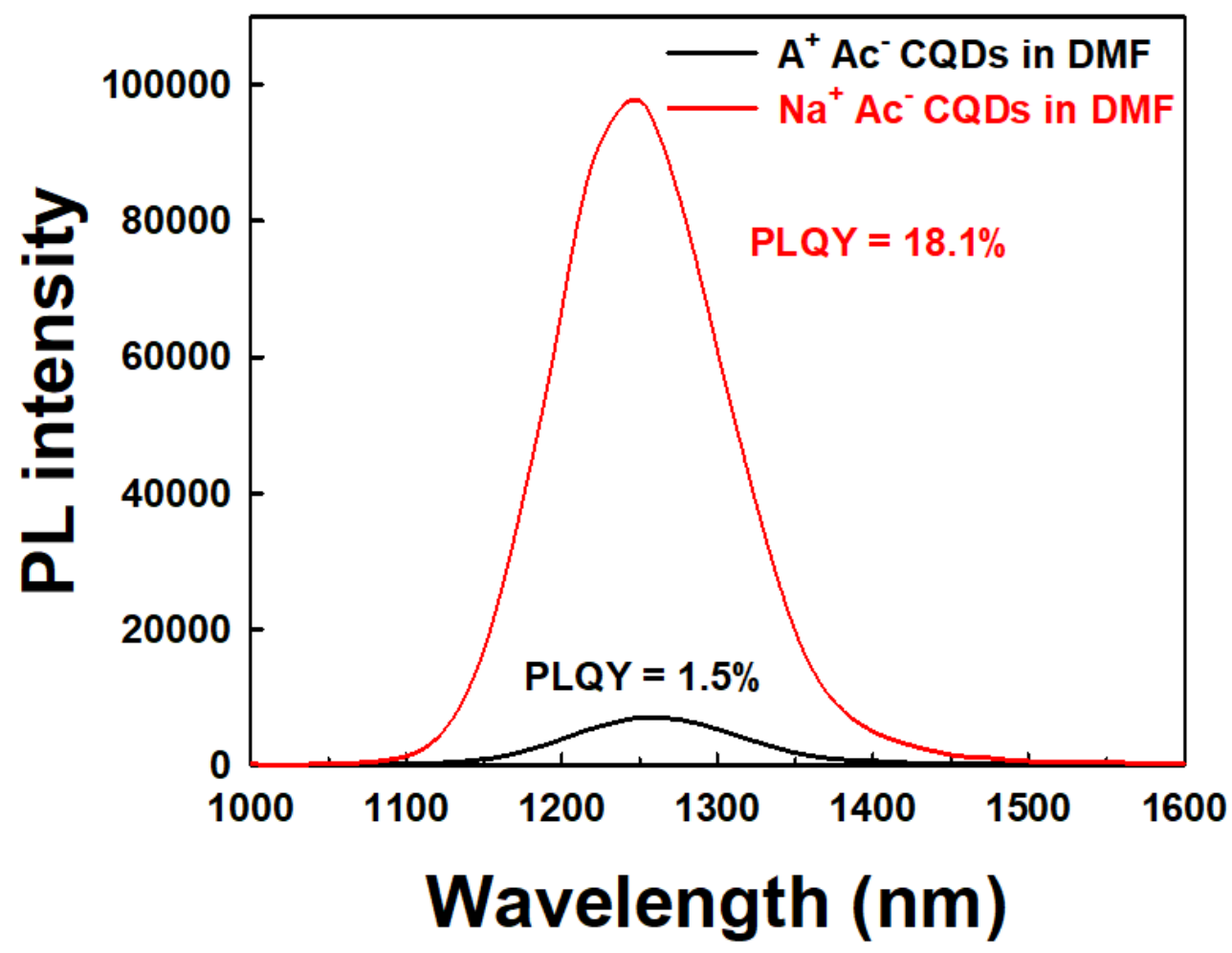

Figure S8. Solution-phase PL spectra and PLQYs of each narrow-bandgap CQD in DMF solvent. 

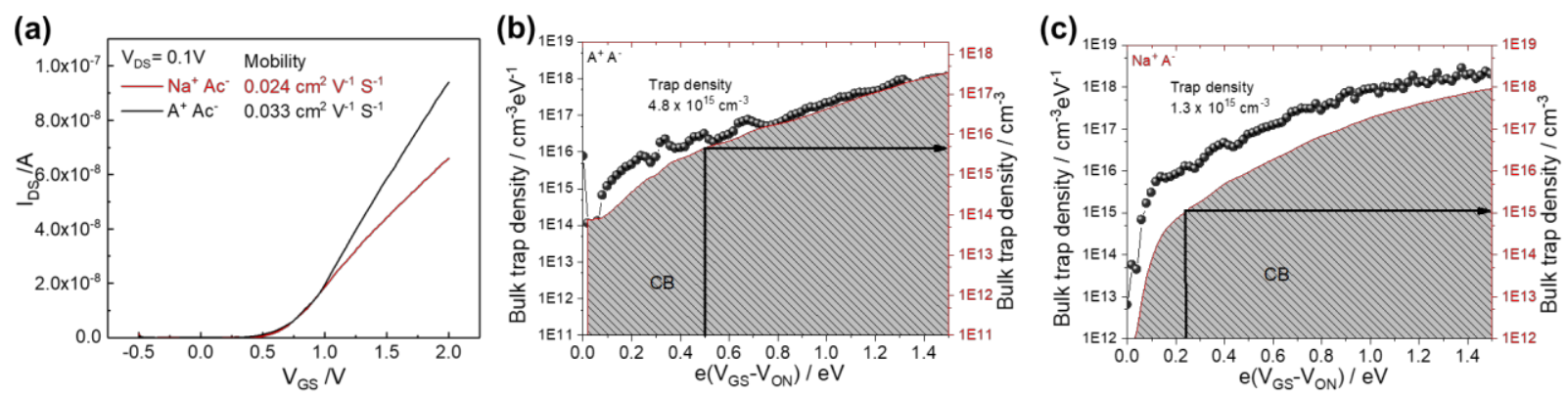

Figure S9. (a) Transfer curves of FET devices fabricated using the lead halide-only and dually-passivated CQDs solids. Trap state densities of (b) lead halide-only and (b) duallypassivated CQDs, calculated from the transfer curves of each FET device. 

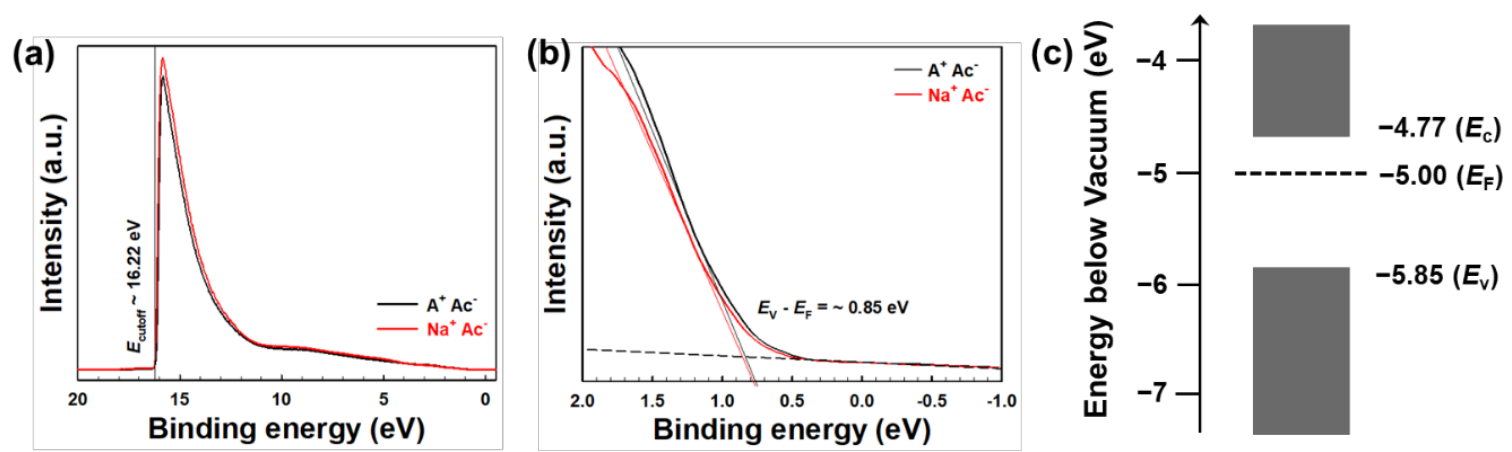

Figure S10. UPS spectra of the lead halide-only and dually-passivated CQDs, measured at (a) high binding energy and (b) low binding energy regions. (c) Energy band diagram of each ligand-exchanged CQD with the $E_{\mathrm{g}}$ of $1.08 \mathrm{eV}$ calculated using UPS results. 


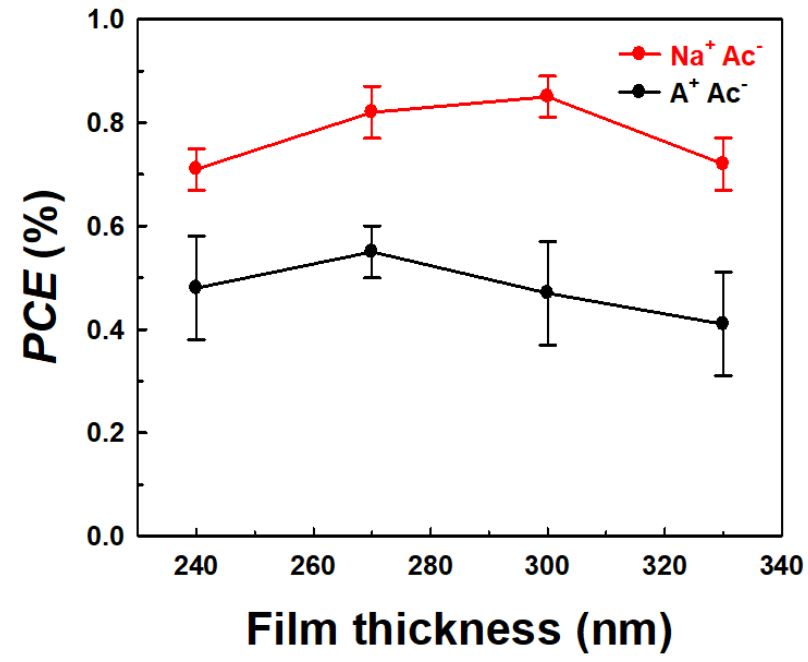

Figure S11. Device performance measured after $1100 \mathrm{~nm}$ long-pass filter of lead halide-only and dually-passivated CQD solar cells with varying the CQD film thickness. 

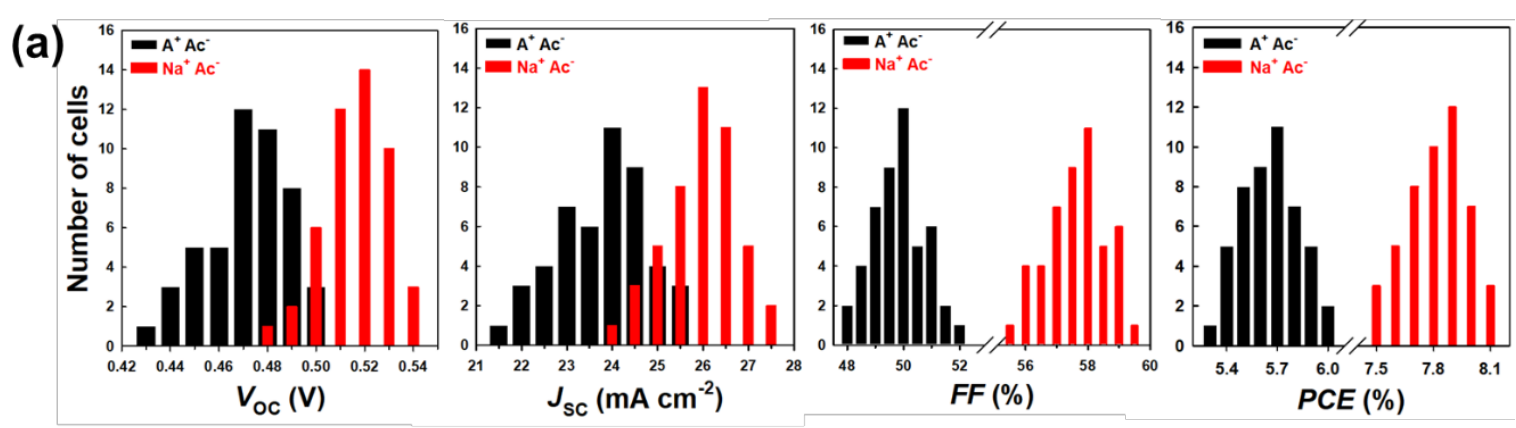

(b)
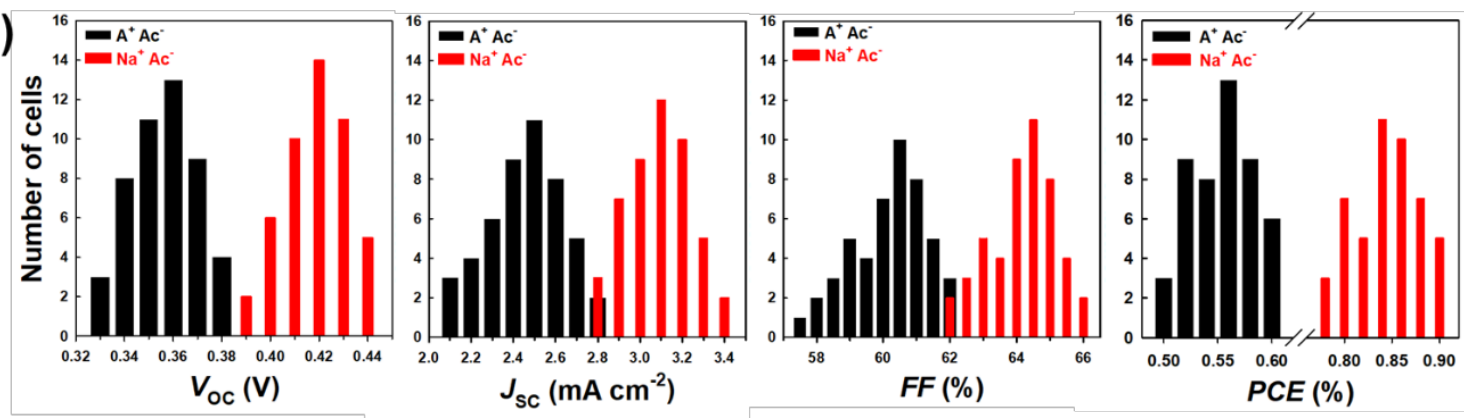

Figure S12. Device histograms for each CQD solar cell, measured (a) at full spectrum and (b) using $1100 \mathrm{~nm}$ long-pass filter. 


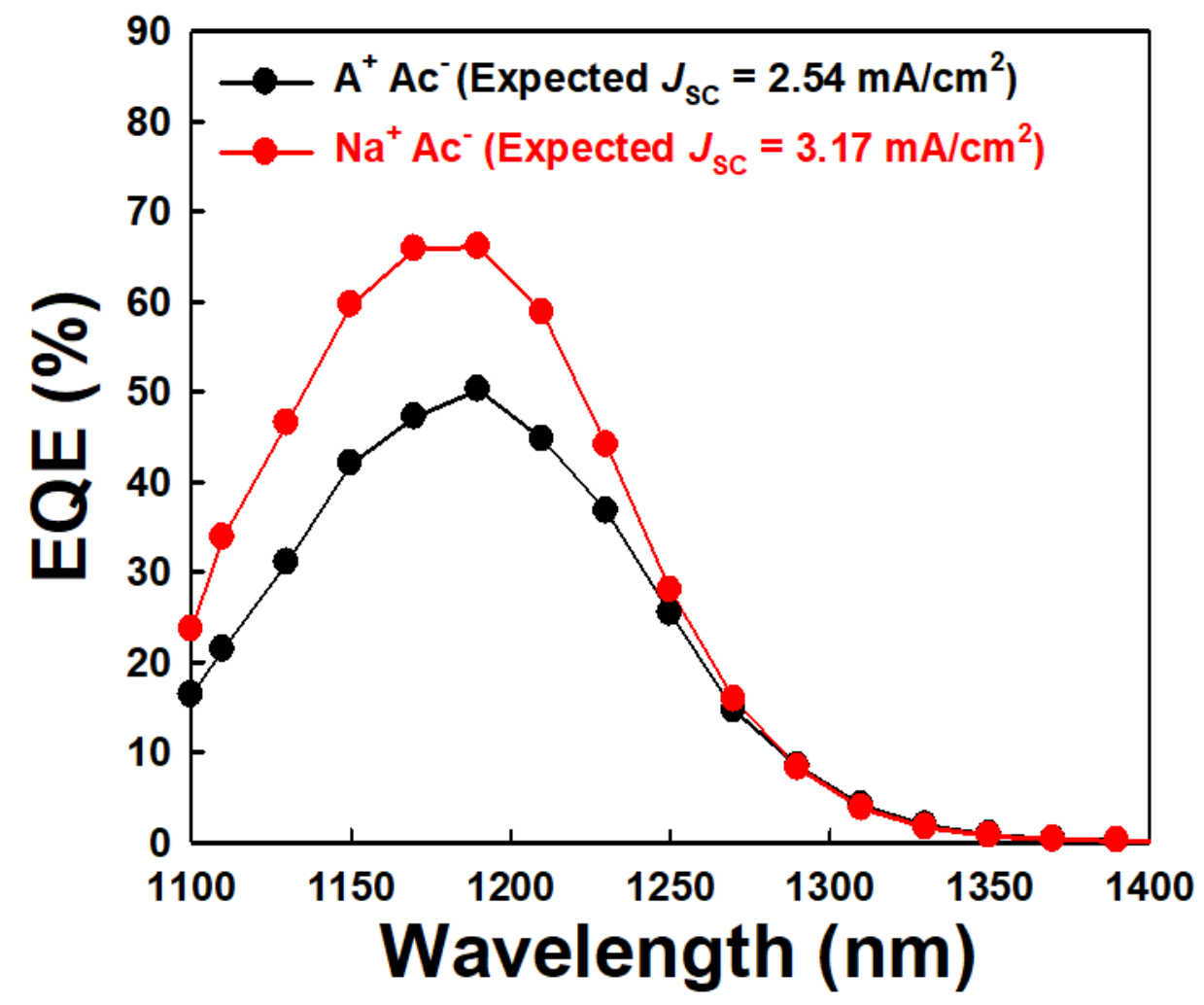

Figure S13. EQE spectra for each solar cell device measured from $1100 \mathrm{~nm}$ to $1400 \mathrm{~nm}$. 

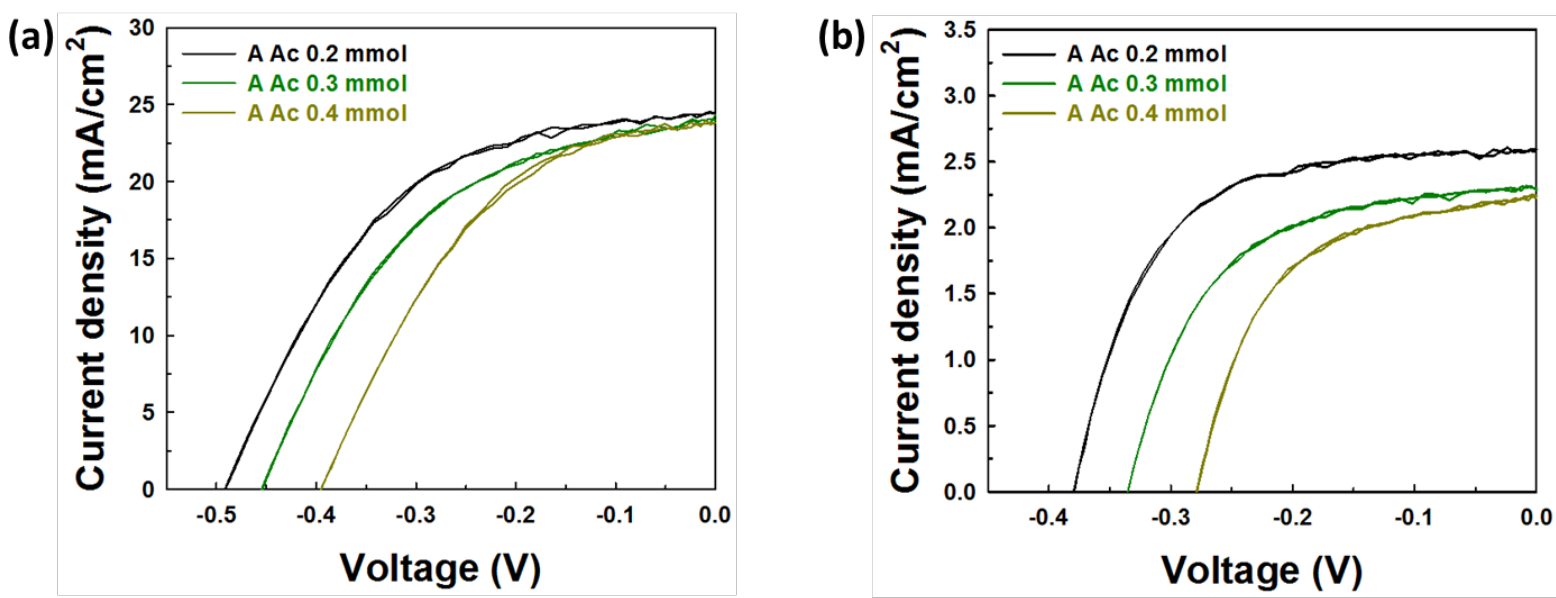

(c) \begin{tabular}{llrrrr}
\hline & & $\mathrm{V}_{\mathrm{oc}}(\mathrm{V})$ & $\mathrm{J}_{\mathrm{sc}}\left(\mathrm{mA} / \mathrm{cm}^{2}\right)$ & FF (\%) & PCE (\%) \\
\hline $\mathrm{A} \cdot \mathrm{Ac}$ & Unfiltered & 0.49 & 24.37 & 50.76 & 6.01 \\
$0.2 \mathrm{mmol}$ & Si filtered $(1100 \mathrm{~nm})$ & 0.38 & 2.60 & 61.29 & 0.60 \\
\hline $\mathrm{A} \cdot \mathrm{Ac}$ & Unfiltered & 0.45 & 24.07 & 47.68 & 5.16 \\
$0.3 \mathrm{mmol}$ & Si filtered $(1100 \mathrm{~nm})$ & 0.34 & 2.31 & 56.33 & 0.44 \\
\hline A $A \mathrm{Ac}$ & Unfiltered & 0.39 & 23.80 & 45.86 & 4.26 \\
$0.4 \mathrm{mmol}$ & Si filtered $(1100 \mathrm{~nm})$ & 0.28 & 2.22 & 55.65 & 0.35 \\
\hline
\end{tabular}

Figure S14. $J-V$ characteristic of solar cell devices fabricated using the lead halide-only passivated CQDs with different amount of ammonium acetate (a) under AM1.5 simulated full solar illumination, (b) after using a silicon solar cell representative filter (long-pass $1100 \mathrm{~nm}$ filter). (c) Device performance parameter with and without long-pass $1100 \mathrm{~nm}$ filter. 


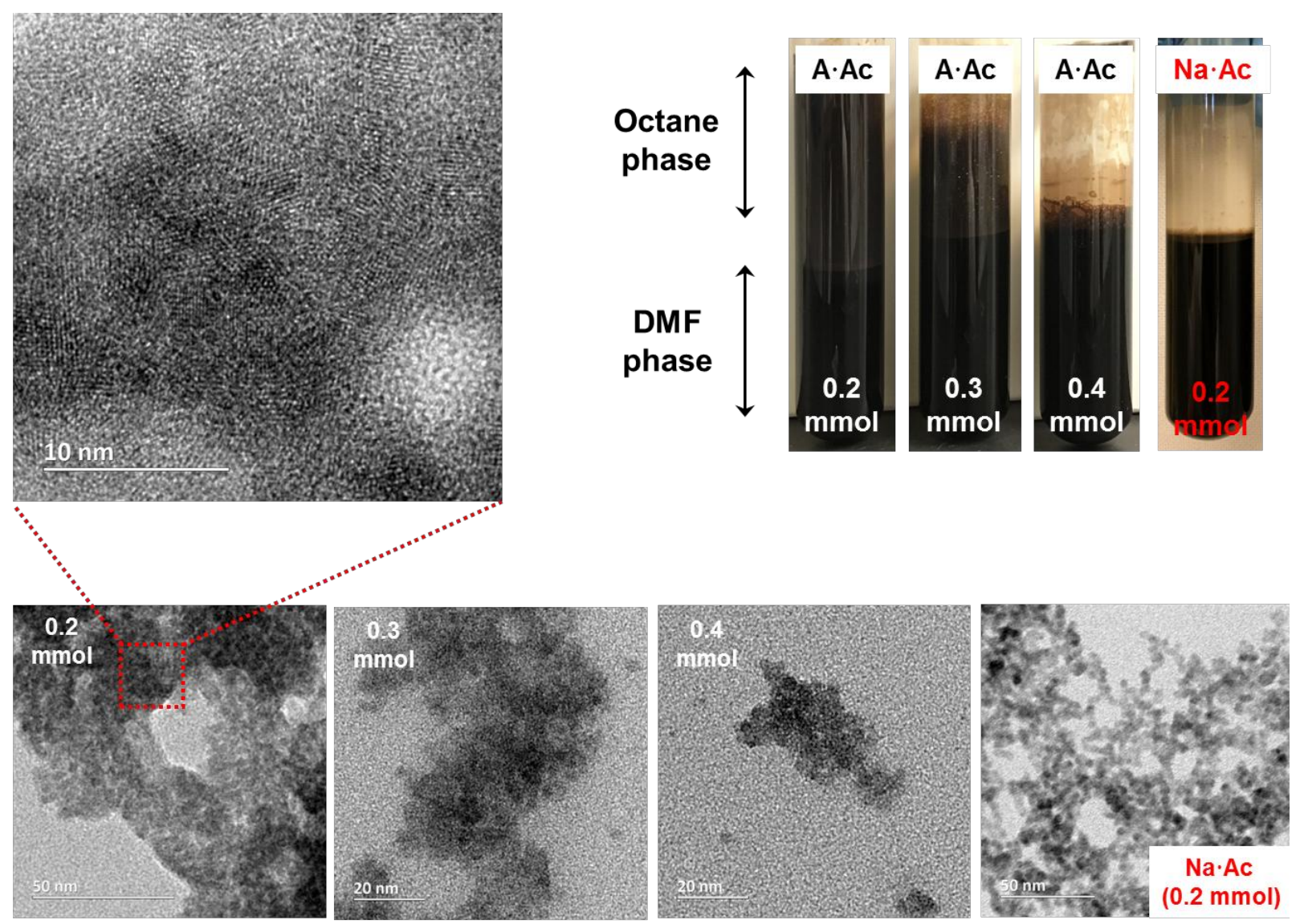

Figure S15. Photo and HR-TEM images of lead halide-only, followed conventional ligand exchange with increasing the amount of ammonium acetate, and dually-passivated with 0.2 mmol sodium acetate CQD solids. HR-TEM images for PbS CQDs were measured using Hitachi HF3300. 


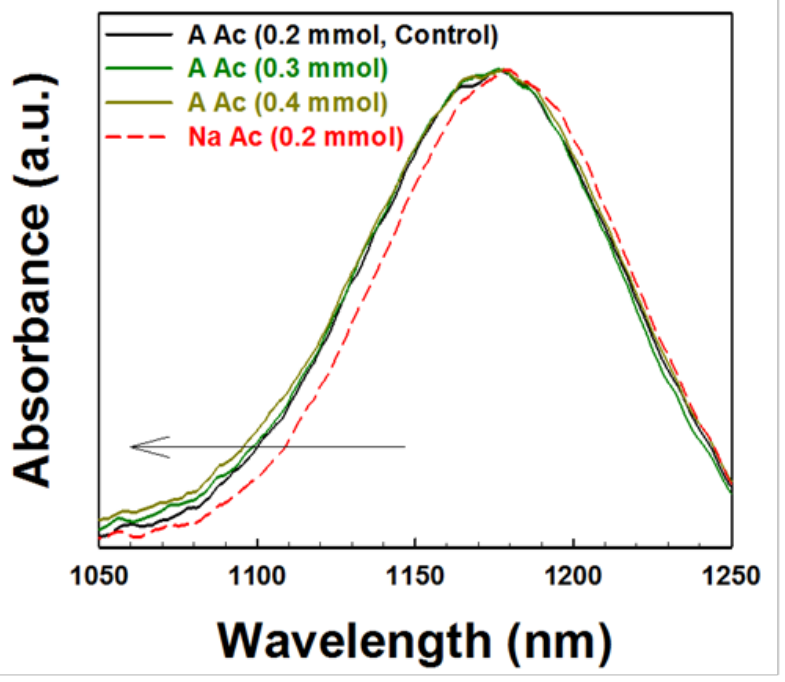

\begin{tabular}{lc}
\hline & FWHM (nm) \\
\hline$A \cdot A c 0.2 \mathrm{mmol}$ & 94 \\
$A \cdot A c 0.3 \mathrm{mmol}$ & 98 \\
$A \cdot A c 0.4 \mathrm{mmol}$ & 101 \\
Na.Ac $0.2 \mathrm{mmol}$ & 84 \\
\hline
\end{tabular}

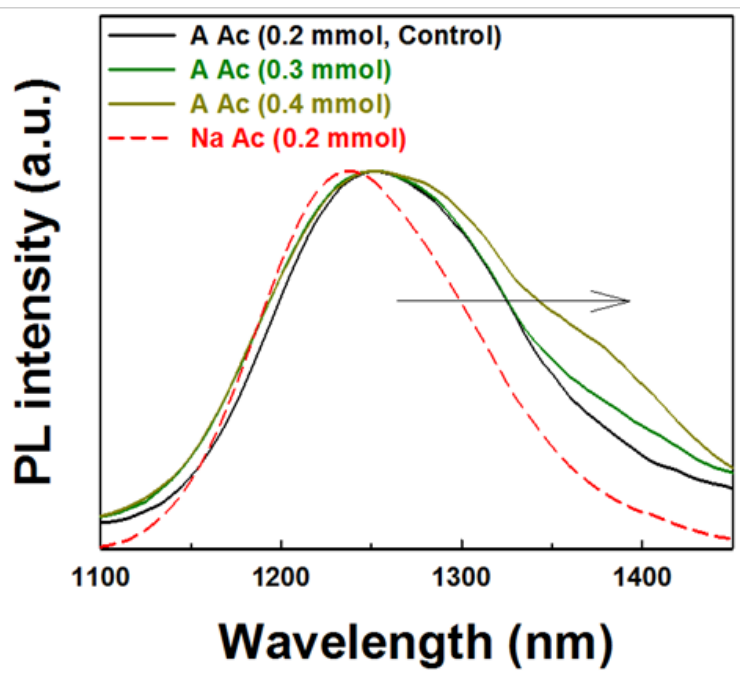

\begin{tabular}{lc}
\hline & FWHM (nm) \\
\hline A.Ac $0.2 \mathrm{mmol}$ & 133 \\
A.Ac $0.3 \mathrm{mmol}$ & 146 \\
$\mathrm{~A} \cdot \mathrm{Ac} 0.4 \mathrm{mmol}$ & 175 \\
$\mathrm{Na} \cdot \mathrm{Ac} 0.2 \mathrm{mmol}$ & 112 \\
\hline
\end{tabular}

Figure S16. Absorbance and PL spectra of lead halide-only with different amount of ammonium acetate and dually-passivated with $0.2 \mathrm{mmol}$ sodium acetate CQD solids. 


\section{References}

1. A. Rolland, J. Richard, J. P. Kleider, D. Mencaraglia, J. Electrochem. Soc. 1993, 140, 3679.

2. G. Kresse, J. Hafner, Phys. Rev. B 1993, 47, 558.

3. G. Kresse, J. Furthmüller, Comput. Mat. Sci. 1996, 6, 15.

4. J. P. Perdew, K. Burke, M. Ernzerhof, Phys. Rev. Lett. 1996, 77, 3865.

5. M. C. Payne, T. A. Arias, J. D. Joannopoulos, Rev. Mod. Phys. 1992, 64, 1045.

6. H. J. Monkhorst, J. D. Pack, Phys. Rev. B 1976, 13, 5188.

7. A. Walsh, J. Phys. Chem. Lett. 2010, 1, 1284.

8. Y. Noda, K. Masumoto, S. Ohba, Y. Saito, K. Toriumi, Y. Iwata, I. Shibuya, Acta Crystallogr., Sect. C: Cryst. Struct. Commun. 1987, 43, 1443. 